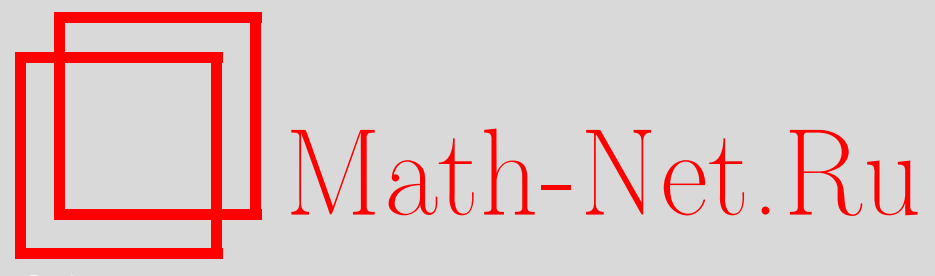

С. И. Митрохин, Об асимптотике спектра дифференциального оператора четного порядка с потенциалом дельта-функцией, Вестн. Сам. гос. техн. ун-та. Сер. Физ.-мат. науки, 2021, номер 4, 634662

DOI: https://doi.org/10.14498/vsgtu1798

Использование Общероссийского математического портала MathNet.Ru подразумевает, что вы прочитали и согласны с пользовательским соглашением

http://www . mathnet.ru/rus/agreement

Параметры загрузки:

IP : 54.210 .77 .194

26 апреля 2023 г., 18:20:43

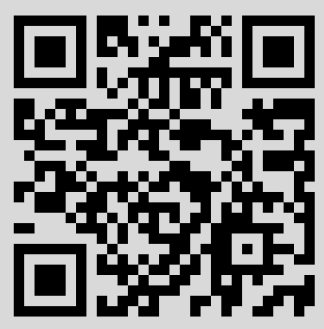


УДК 517.927

\title{
Об асимптотике спектра дифференциального оператора четного порядка с потенциалом дельта-функцией
}

\section{С. И. Митрохин}

Московский государственный университет имени М. В. Ломоносова, Научно-исследовательский вычислительный центр, Россия, 119991, Москва, Ленинские горы, 1, стр. 4.

\begin{abstract}
Аннотация
Изучается последовательность дифференциальных операторов высокого четного порядка, потенциалы которых сходятся к дельта-функции Дирака. Рассматривается один из видов разделённых граничных условий. В точках разрыва потенциала необходимо изучить условия склейки для корректного определения решений соответствующих дифференциальных уравнений. При больших значениях спектрального параметра методом Наймарка выписаны асимптотические решения дифференциальных уравнений. Изучены условия склейки, исследованы граничные условия, выведено уравнение на собственные значения рассматриваемого дифференциального оператора. Методом последовательных приближений найдена асимптотика спектра изучаемых дифференциальных операторов, предел которой задаёт спектр оператора с потенциалом дельта-функцией.
\end{abstract}

Ключевые слова: дифференциальный оператор, дельта-функция Дирака, асимптотика решений дифференциального уравнения, кусочногладкий потенциал, собственные значения, асимптотика спектра.

Получение: 15 июля 2020 г. / Исправление: 23 ноября 2021 г. / Принятие: 6 декабря 2021 г. / Публикация онлайн: 29 декабря 2021 г.

\section{Научная статья}

(C) Коллектив авторов, 2021

(C) СамГТУ, 2021 (составление, дизайн, макет)

(2) (9) Контент публикуется на условиях лицензии Creative Commons Attribution 4.0 International (https://creativecommons.org/licenses/by/4.0/deed.ru)

\section{Образец для цитирования}

Митрохин С. И. Об асимптотике спектра дифференциального оператора четного порядка с потенциалом дельта-функцией // Вестн. Сам. гос. техн. ун-та. Сер. Физ.-мат. науки, 2021. Т. 25, № 4. C. 634-662. https://doi.org/10.14498/vsgtu1798.

Сведения об авторе

Сергей Иванович Митрохин (1) https://orcid.org/0000-0003-1896-0563

кандидат физико-математических наук, доцент; старший научный сотрудник; научноисследовательский вычислительный центр; e-mail: mitrokhin-sergey@yandex.ru 
Введение и исторический обзор. Дифференциальный оператор, потенциалом которого является дельта-функция, можно рассматривать как предел последовательности операторов с кусочно-гладкими потенциалами. Развитие спектральной теории дифференциальных операторов идет в сторону уменьшения гладкости коэффициентов дифференциальных уравнений, задающих эти операторы. Различные вопросы спектральной теории операторов второго порядка с негладкими коэффициентами (с кусочно-гладкими потенциалами либо кусочно-гладкой весовой функцией) были рассмотрены в работах [1-5].

В работе [6] были найдены асимптотики собственных значений дифференциальных операторов второго порядка с суммируемым потенциалом. В работах [7-9] были изучены спектральные свойства операторов четвертого, шестого и выше порядков с разделенными граничными условиями, а в работе [10] - с неразделенными граничными условиями с суммируемым на отрезке потенциалом.

В работах [11-13] рассматривались различные операторы второго порядка с сингулярными потенциалами, в том числе и операторы с потенциалом дельта-функцией. Необходимость изучения операторов, потенциалом которых является дельта-функция Дирака, следует из богатства их физических приложений, примеры которых изложены в работах [14-18].

Во всех этих работах рассматривались операторы второго порядка. Операторы порядка выше второго, потенциалами которых является дельта-функция, до сих пор не изучались.

1. Постановка задачи. Изучим дифференциальный оператор с кусочногладкими коэффициентами, задаваемый на отрезке $[0 ; \pi]$ дифференциальными уравнениями

$$
\begin{array}{ll}
y_{1}^{(8)}(x)+q_{1}(x) y_{1}(x)=\lambda a^{8} y_{1}(x), & 0 \leqslant x<x_{1 n} ; \\
y_{2}^{(8)}(x)+q_{2}(x) y_{2}(x)=\lambda a^{8} y_{2}(x), & x_{1 n} \leqslant x \leqslant x_{0} \\
y_{3}^{(8)}(x)+q_{3}(x) y_{3}(x)=\lambda a^{8} y_{3}(x), & x_{0}<x \leqslant x_{2 n} ; \\
y_{4}^{(8)}(x)+q_{4}(x) y_{4}(x)=\lambda a^{8} y_{4}(x), & x_{2 n}<x \leqslant \pi,
\end{array}
$$

где $\lambda \in \mathbb{C}$ - спектральный параметр; $\rho(x)=a^{8}$ - весовая функция, $a>0$; $Q_{n}(x)=q_{1}(x) \vee q_{2}(x) \vee q_{3}(x) \vee q_{4}(x)$ - потенциал, на который накладываются следующие условия:

$$
\begin{aligned}
& q_{1}(x)=0, \quad x \in\left[0, x_{1 n}\right] ; \quad q_{4}(x)=0, \quad x \in\left(x_{2 n}, \pi\right] \\
& x_{1 n}=x_{0}-\frac{k_{0}}{n}, \quad k_{0}>0 ; \quad x_{2 n}=x_{0}+\frac{m_{0}}{n}, \quad m_{0}>0 ; \\
& q_{2}(x) \in C^{7}\left[x_{1 n} ; x_{0}\right] ; \quad q_{3}(x) \in C^{7}\left(x_{0}, x_{2 n}\right] ; \\
& \quad \lim _{x \rightarrow x_{1 n}+0} q_{2}(x)=0 ; \quad \lim _{x \rightarrow x_{0}-0} q_{2}(x)=+\infty ; \\
& \lim _{x \rightarrow x_{0}+0} q_{3}(x)=+\infty ; \quad \lim _{x \rightarrow x_{2 n}-0} q_{3}(x)=0 ; \\
& \int_{x_{1 n}}^{x_{0}} q_{2}(t) d t=H_{1 n} ; \quad \int_{x_{0}}^{x_{2 n}} q_{3}(t) d t=H_{2 n} ; \quad H_{1 n}+H_{2 n}=1 .
\end{aligned}
$$


В точках $x_{1 n}, x_{0}, x_{2 n}$ разрыва коэффициентов потребуем выполнения следующих условий склейки:

$$
\begin{aligned}
y_{1}\left(x_{1 n}-0\right)=y_{2}\left(x_{1 n}+0\right) ; & y_{1}^{(m)}\left(x_{1 n}-0\right)=y_{2}^{(m)}\left(x_{1 n}+0\right), \quad m=1,2, \ldots, 7 ;(6) \\
y_{2}\left(x_{0}-0\right)=y_{3}\left(x_{0}+0\right) ; & y_{2}^{(m)}\left(x_{0}-0\right)=y_{3}^{(m)}\left(x_{0}+0\right), \quad m=1,2, \ldots, 7 ; \quad(7) \\
y_{3}\left(x_{2 n}-0\right)=y_{4}\left(x_{2 n}+0\right) ; & y_{3}^{(m)}\left(x_{2 n}-0\right)=y_{4}^{(m)}\left(x_{2 n}+0\right), \quad m=1,2, \ldots, 7 .(8)
\end{aligned}
$$

Будем рассматривать граничные условия вида

$$
\begin{aligned}
& y_{1}^{\left(m_{1}\right)}(0)=y_{1}^{\left(m_{2}\right)}(0)=\cdots=y_{1}^{\left(m_{6}\right)}(0)=y_{4}^{\left(n_{1}\right)}(\pi)=y_{4}^{\left(n_{2}\right)}(\pi)=0, \\
& m_{1}<m_{2}<\cdots<m_{6}, n_{1}<n_{2} ; m_{k}, n_{1}, n_{2} \in\{0,1,2, \ldots, 7\}, k=1,2, \ldots, 6 .
\end{aligned}
$$

Из условий (5) следует, что потенциал $Q_{n}(x)$ удовлетворяет следующим условиям:

$$
\begin{aligned}
& \lim _{n \rightarrow+\infty} Q_{n}(x)=\delta\left(x-x_{0}\right)=\left[\begin{array}{cl}
0, & x \in\left[0 ; x_{0}\right) \\
+\infty, & x=x_{0} \\
0, & x \in\left(x_{0} ; \pi\right]
\end{array}\right. \\
& \int_{0}^{\pi} Q_{n}(x) d x=\int_{0}^{\pi} \delta\left(x-x_{0}\right) d x=H_{1 n}+H_{2 n}=1 .
\end{aligned}
$$

Поэтому мы фактически изучим асимптотику спектра оператора, задаваемого дифференциальным уравнением

$$
y^{(8)}(x)+\delta\left(x-x_{0}\right) y(x)=\lambda a^{8} y(x), \quad 0 \leqslant x \leqslant \pi, \quad a>0,
$$

с граничными условиями (9).

2. Асимптотика решений дифференциальных уравнений (1)-(4) при больших значениях спектрального параметра $\boldsymbol{\lambda}$. Пусть $\lambda=s^{8}$, $s=\sqrt[8]{\lambda}$, при этом для корректности дальнейших вычислений зафиксируем ту ветвь арифметического корня восьмой степени, для которой $\sqrt[8]{1}=+1$. Обозначим через $\omega_{k}(k=1,2, \ldots, 8)$ различные корни восьмой степени из единицы:

$$
\begin{aligned}
& \omega_{k}^{8}=1 ; \quad \omega_{k}=e^{\frac{2 \pi i}{8}(k-1)}, \quad k=1,2, \ldots, 8 ; \\
& \omega_{1}=1, \quad \omega_{2}=e^{\frac{2 \pi i}{8}}=\frac{\sqrt{2}}{2}+\frac{\sqrt{2} i}{2}=z \neq 0 ; \\
& \omega_{3}=\omega_{2}^{2}=e^{\frac{4 \pi i}{8}}=i, \quad \omega_{4}=-\frac{\sqrt{2}}{2}+\frac{\sqrt{2} i}{2} \\
& \omega_{5}=-1, \quad \omega_{6}=-\frac{\sqrt{2}}{2}-\frac{\sqrt{2} i}{2}=\bar{\omega}_{4}, \\
& \omega_{7}=-i=\bar{\omega}_{3}, \quad \omega_{8}=\frac{\sqrt{2}}{2}-\frac{\sqrt{2} i}{2}=\bar{\omega}_{2} ; \\
& \omega_{m}=z^{m-1}, \quad m=1,2, \ldots, 8 .
\end{aligned}
$$


Числа $\omega_{k}(k=1,2, \ldots, 8)$ из $(10)$ делят единичную окружность на восемь равных частей и для них справедливы следующие свойства:

$$
\begin{gathered}
\sum_{k=1}^{8} \omega_{k}^{m}=0, \quad m=1,2, \ldots, 7 ; \quad \sum_{k=1}^{8} \omega_{k}^{m}=8, \quad m=0, m=8 ; \\
\quad \sum_{k=0}^{7} \omega_{m}^{k}=0, \quad m=2,3, \ldots, 8 ; \quad \sum_{k=0}^{7} \omega_{m}^{k}=8, \quad m=1 .
\end{gathered}
$$

Аналогично монографии [19, гл. 2] устанавливаются следующие утверждения.

Теорема 1. Общее решение дифберенциального уравнения $(1)\left(q_{1}(x)=0\right.$ при $\left.x \in\left[0 ; x_{1 n}\right)\right)$ имеет вид

$$
\begin{aligned}
& y_{1}(x, s)=\sum_{k=1}^{8} C_{1 k} y_{1 k}(x, s) \\
& y_{1}^{(m)}(x, s)=\sum_{k=1}^{8} C_{1 k} y_{1 k}^{(m)}(x, s), \quad m=1,2, \ldots, 7,
\end{aligned}
$$

где $C_{1 k}$ - произволъные постоянные, $k=1,2, \ldots, 8$;

$$
\begin{array}{r}
y_{1 k}(x, s)=e^{a \omega_{k} s x}, \quad y_{1 k}^{(m)}(x, s)=\left(a \omega_{k} s\right)^{m} e^{a \omega_{k} s x}, \\
k=1,2, \ldots, 8, \quad m=1,2, \ldots, 7 .
\end{array}
$$

Теорема 2. Общее решение дифференииалъного уравнения (2) представляется в виде

$$
\begin{aligned}
& y_{2}(x, s)=\sum_{k=1}^{8} C_{2 k} y_{2 k}(x, s) \\
& y_{2}^{(m)}(x, s)=\sum_{k=1}^{8} C_{2 k} y_{2 k}^{(m)}(x, s), \quad x_{1 n} \leqslant x \leqslant x_{0}, \quad m=1,2, \ldots, 7,
\end{aligned}
$$

әде $C_{2 k}$ - произвольные постоянные, $k=1,2, \ldots, 8$, при этом для фундаментальной системы решений $\left\{y_{2 k}(x, s)\right\}_{k=1}^{8}$ справедливы следующие асимптотические формуль и оченки:

$$
\begin{gathered}
y_{2 k}(x, s)=e^{a \omega_{k} s x}\left[1+\frac{\omega_{k} A_{7}(x)}{s^{7}}+\frac{A_{8}^{0}(x)}{s^{8}}+\underline{O}\left(\frac{e^{|\operatorname{Im} s| a x}}{s^{9}}\right)\right], \\
k=1,2, \ldots, 8 ; \\
y_{2 k}^{(m)}(x, s)=\left(a \omega_{k} s\right)^{m} e^{a \omega_{k} s x}\left[1+\frac{\omega_{k} A_{7}(x)}{s^{7}}+\frac{A_{8}^{m}(x)}{s^{8}}+\underline{O}\left(\frac{e^{|\operatorname{Im} s| a x}}{s^{9}}\right)\right], \\
k=1,2, \ldots, 8, \quad m=1,2, \ldots, 7 \\
A_{7}(x)=-\frac{1}{8 a^{7}} \int_{x_{1 n}}^{x} q_{2}(t) d t ; \quad A_{7}\left(x_{1 n}\right)=0 ; \quad A_{7}^{\prime}(x)=-\frac{q_{2}(x)}{8 a^{7}}
\end{gathered}
$$




$$
\begin{gathered}
A_{8}^{0}(x)=\frac{7 q_{2}(x)-7 q_{2}\left(x_{1 n}\right)}{16 a^{8}} ; \quad A_{8}^{0}\left(x_{1 n}\right)=0 ; \quad A_{8}^{1}(x)=\frac{5 q_{2}(x)-7 q_{2}\left(x_{1 n}\right)}{16 a^{8}} ; \\
A_{8}^{m}(x)=\frac{(7-2 m) q_{2}(x)-7 q_{2}\left(x_{1 n}\right)}{16 a^{8}}, \quad m=0,1,2, \ldots, 7 ; \\
A_{8}^{7}(x)=\frac{-7 q_{2}(x)-7 q_{2}\left(x_{1 n}\right)}{16 a^{8}} ; \\
\sum_{k=0}^{7} A_{8}^{k}(x)=\sum_{k=0}^{7} A_{8}^{k}\left(x_{1 n}\right)=\sum_{k=0}^{7} A_{8}^{k}\left(x_{0}\right)=D_{8}=\frac{-7 q_{2}\left(x_{1 n}\right)}{2 a^{8}} .
\end{gathered}
$$

Теорема 3. Общее решение дифференциального уравнения (3) имеет вид

$$
\begin{aligned}
& y_{3}(x, s)=\sum_{k=1}^{8} C_{3 k} y_{3 k}(x, s) \\
& y_{3}^{(m)}(x, s)=\sum_{k=1}^{8} C_{3 k} y_{3 k}^{(m)}(x, s), \quad m=1,2, \ldots, 7, \quad x_{0}<x \leqslant x_{2 n},
\end{aligned}
$$

где $C_{3 k}-$ произвольные постолнные, $k=1,2, \ldots, 8$;

$$
\begin{gathered}
y_{3 k}(x, s)=e^{a \omega_{k} s x}\left[1+\frac{\omega_{k} B_{7}(x)}{s^{7}}+\frac{B_{8}^{0}(x)}{s^{8}}+\underline{O}\left(\frac{e^{|\operatorname{Im} s| a x}}{s^{9}}\right)\right], \\
k=1,2, \ldots, 8 ; \\
y_{3 k}^{(m)}(x, s)=\left(a \omega_{k} s\right)^{m} e^{a \omega_{k} s x}\left[1+\frac{\omega_{k} B_{7}(x)}{s^{7}}+\frac{B_{8}^{m}(x)}{s^{8}}+\underline{O}\left(\frac{e^{|\mathrm{Im} s| a x}}{s^{9}}\right)\right], \\
B_{7}(x)=-\frac{1}{8 a^{7}} \int_{x_{0}}^{x} q_{3}(t) d t ; \quad B_{7}\left(x_{0}\right)=0 ; \quad B_{7}^{\prime}(x)=-\frac{q_{3}(x)}{8 a^{7}} ; \\
B_{8}^{0}(x)=\frac{7 q_{3}(x)-7 q_{3}\left(x_{0}\right)}{16 a^{8}} ; \quad B_{8}^{0}\left(x_{0}\right)=0 ; \quad B_{8}^{1}(x)=\frac{5 q_{3}(x)-7 q_{3}\left(x_{0}\right)}{16 a^{8}} ; \\
B_{8}^{m}(x)=\frac{(7-2 m) q_{3}(x)-7 q_{3}\left(x_{0}\right)}{16 a^{8}}, \quad m=0,1,2, \ldots, 7 ; \\
B_{8}^{7}(x)=\frac{-7 q_{3}(x)-7 q_{3}\left(x_{0}\right)}{16 a^{8}} ; \\
\sum_{k=0}^{7} B_{8}^{k}(x)=\sum_{k=0}^{7} B_{8}^{k}\left(x_{2 n}\right)=E_{8}=\frac{-7 q_{3}\left(x_{0}\right)}{2 a^{8}} .
\end{gathered}
$$

Теорема 4. Общее решение дифберенциального уравнения $(4)\left(q_{4}(x)=0\right.$ при $\left.x \in\left(x_{2 n} ; \pi\right)\right)$ представляется в виде

$$
\begin{aligned}
& y_{4}(x, s)=\sum_{k=1}^{8} C_{4 k} y_{4 k}(x, s) ; \\
& y_{4}^{(m)}(x, s)=\sum_{k=1}^{8} C_{4 k} y_{4 k}^{(m)}(x, s), \quad m=1,2, \ldots, 7,
\end{aligned}
$$


где $C_{4 k}-$ произвольные постоянные, $k=1,2, \ldots, 8$;

$$
\begin{array}{r}
y_{4 k}(x, s)=e^{a \omega_{k} s x}, \quad y_{4 k}^{(m)}(x, s)=\left(a \omega_{k} s\right)^{m} e^{a \omega_{k} s x}, \\
k=1,2, \ldots, 8, \quad m=1,2, \ldots, 7 .
\end{array}
$$

Обозначим через $\Delta_{00}$ - определитель Вандермонда чисел $\omega_{1}, \omega_{2}, \ldots, \omega_{8}$ из (10)-(12):

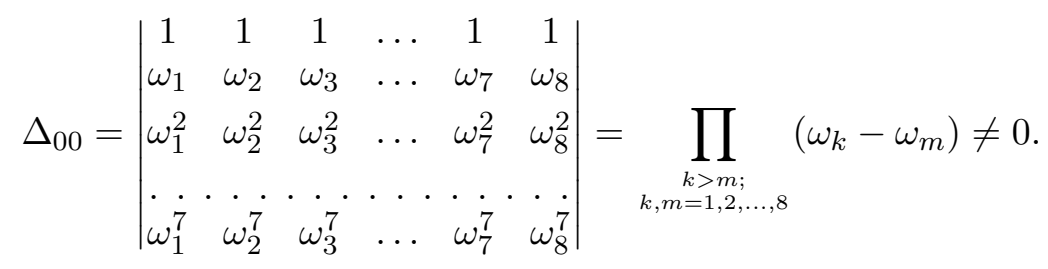

Справедливо следующее утверждение.

Теорема 5. Пусть $\left(\delta_{m k}\right)(m, k=1,2, \ldots, 8)$ - матрии,а алгебрачческих миноров к элементам $b_{m k}(m, k=1,2, \ldots, 8)$ определителя $\Delta_{00}$ из (29). Тогда

$$
\begin{aligned}
\left(\delta_{m k}\right)= & \left(\begin{array}{cccc}
\delta_{11} & \delta_{12} & \ldots & \delta_{18} \\
\delta_{21} & \delta_{22} & \ldots & \delta_{28} \\
\ldots & \ldots & \ldots & . \\
\delta_{71} & \delta_{72} & \ldots & \delta_{78} \\
\delta_{81} & \delta_{82} & \ldots & \delta_{88}
\end{array}\right)= \\
& =\frac{\Delta_{00}}{8}\left(\begin{array}{ccccccc}
1 & -1 & 1 & -1 & \ldots & 1 & -1 \\
-\omega_{1}^{-1} & \omega_{2}^{-1} & -\omega_{3}^{-1} & \omega_{4}^{-1} & \ldots & -\omega_{7}^{-1} & \omega_{8}^{-1} \\
\omega_{1}^{-2} & -\omega_{2}^{-2} & \omega_{3}^{-2} & -\omega_{4}^{-2} & \ldots & \omega_{7}^{-2} & -\omega_{8}^{-2} \\
\ldots & \ldots & \ldots & \ldots & & \\
\omega_{1}^{-6} & -\omega_{2}^{-6} & \omega_{3}^{-6} & -\omega_{4}^{-6} & \ldots & \omega_{7}^{-6} & -\omega_{8}^{-6} \\
-\omega_{1}^{-7} & \omega_{2}^{-7} & -\omega_{3}^{-7} & \omega_{4}^{-7} & \ldots & -\omega_{7}^{-7} & \omega_{8}^{-7}
\end{array}\right) .
\end{aligned}
$$

Доказательство теоремы 5 можно найти в работе [20].

3. Изучение условий склейки (8). С помощью формул (21) и (27) из условий склейки (8) получим следующую систему уравнений:

$$
\left\{\begin{array}{c}
y_{4}\left(x_{2 n}+0, s\right) \stackrel{(8)}{=} y_{3}\left(x_{2 n}-0, s\right) \Leftrightarrow \sum_{k=1}^{8} C_{4 k} y_{4 k}\left(x_{2 n}+0, s\right)= \\
=\sum_{k=1}^{8} C_{3 k} y_{3 k}\left(x_{2 n}-0, s\right) ; \\
\frac{y_{4}^{(m)}\left(x_{2 n}+0, s\right)}{(a s)^{m}} \stackrel{(8)}{=} \frac{y_{3}^{(m)}\left(x_{2 n}-0, s\right)}{(a s)^{m}} \Leftrightarrow \sum_{k=1}^{8} C_{4 k} \frac{y_{4 k}^{(m)}\left(x_{2 n}+0, s\right)}{(a s)^{m}}= \\
=\sum_{k=1}^{8} C_{3 k} \frac{y_{3 k}^{(m)}\left(x_{2 n}-0, s\right)}{(a s)^{m}}, \quad m=1,2, \ldots, 7 .
\end{array}\right.
$$


Из метода Крамера следует, что решение системы (31) имеет следующий вид:

$$
C_{41}=\frac{\Delta_{41}}{\Delta_{04}(s) \neq 0}, \quad C_{42}=\frac{\Delta_{42}}{\Delta_{04}(s) \neq 0}, \quad \ldots, \quad C_{48}=\frac{\Delta_{48}}{\Delta_{04}(s) \neq 0}
$$

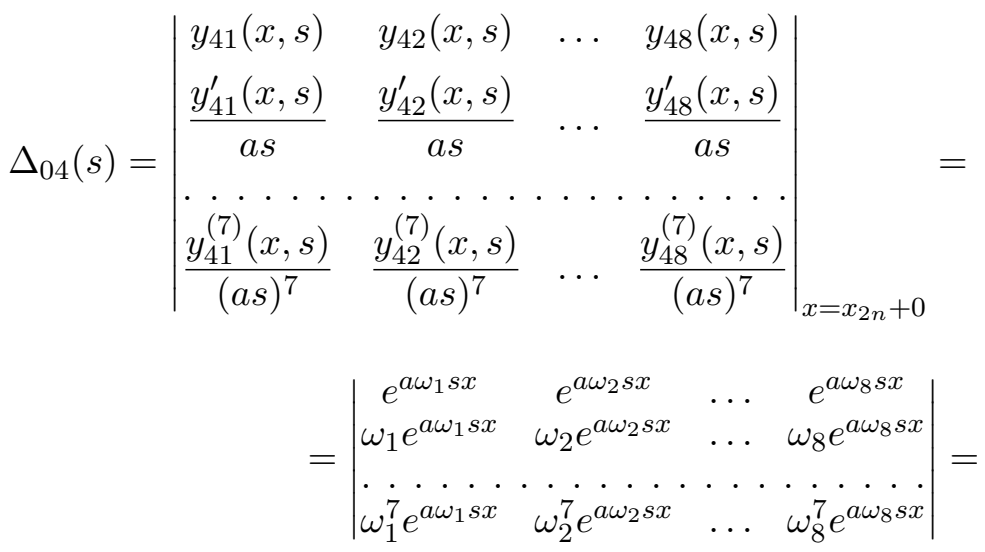

$$
\begin{aligned}
& =e^{a\left(\omega_{1}+\omega_{2}+\cdots+\omega_{8}\right) s x} \Delta_{00} \stackrel{(11)}{=} e^{0} \Delta_{00}=\Delta_{00} \neq 0 .
\end{aligned}
$$

Определители $\Delta_{4 k}(k=1,2, \ldots, 8)$ из формулы (32) получаются из определителя $\Delta_{04}(s)$ из $(33)$ заменой $k$-го столбца на столбец

$$
\left(\sum_{k=1}^{8} C_{3 k} y_{3 k}\left(x_{2 n}-0, s\right) \sum_{k=1}^{8} C_{3 k} \frac{y_{3 k}^{\prime}\left(x_{2 n}-0, s\right)}{a s} \cdots \sum_{k=1}^{8} C_{3 k} \frac{y_{3 k}^{(7)}\left(x_{2 n}-0, s\right)}{(a s)^{7}}\right)^{\top} .
$$

Таким образом, из (32)-(34) следует, что определитель $\Delta_{41}$ выписывается в следующем виде:

$$
\begin{aligned}
& \Delta_{41}=\left|\begin{array}{cccc}
\sum_{k=1}^{8} C_{3 k} y_{3 k}\left(x_{2 n}-0, s\right) & e^{a \omega_{2} s x_{2 n}} & \ldots & e^{a \omega_{8} s x_{2 n}} \\
\sum_{k=1}^{8} C_{3 k} \frac{y_{3 k}^{\prime}\left(x_{2 n}-0, s\right)}{a s} & \omega_{2} e^{a \omega_{2} s x_{2 n}} & \ldots & \omega_{8} e^{a \omega_{8} s x_{2 n}} \\
\ldots \ldots \ldots \ldots & \ldots \ldots \ldots & \ldots \ldots \ldots \\
\sum_{k=1}^{8} C_{3 k} \frac{y_{3 k}^{(7)}\left(x_{2 n}-0, s\right)}{(a s)^{7}} & \omega_{2}^{7} e^{a \omega_{2} s x_{2 n}} & \ldots & \omega_{8}^{7} e^{a \omega_{8} s x_{2 n}}
\end{array}\right|= \\
& =e^{a \omega_{2} s x_{2 n}} e^{a \omega_{3} s x_{2 n}}(\ldots) e^{a \omega_{8} s x_{2 n}} \sum_{k=1}^{8} C_{3 k} \Delta_{41 k}= \\
& =C_{31} \Delta_{411}+C_{32} \Delta_{412}+\cdots+C_{38} \Delta_{418} \text {; }
\end{aligned}
$$




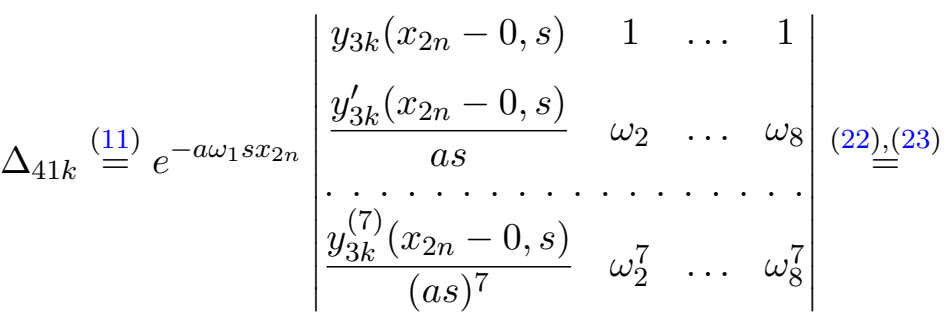

$$
\begin{aligned}
& =e^{-a \omega_{1} s x_{2 n}} e^{a \omega_{k} s x_{2 n}}\left|\begin{array}{lllll}
1 \cdot\left[1+\frac{\omega_{k} B_{7}\left(x_{2 n}\right)}{s^{7}}+\frac{B_{8}^{0}\left(x_{2 n}\right)}{s^{8}}+\ldots\right] & 1 & \ldots & 1 \\
\omega_{k}\left[1+\frac{\omega_{k} B_{7}\left(x_{2 n}\right)}{s^{7}}+\frac{B_{8}^{1}\left(x_{2 n}\right)}{s^{8}}+\ldots\right] & \omega_{2} & \ldots & \omega_{8} \\
\ldots \ldots \ldots & \ldots \ldots & \ldots & \ldots & \ldots \\
\omega_{k}^{7}\left[1+\frac{\omega_{k} B_{7}\left(x_{2 n}\right)}{s^{7}}+\frac{B_{8}^{7}\left(x_{2 n}\right)}{s^{8}}+\ldots\right] & \omega_{2}^{7} & \ldots & \omega_{8}^{7}
\end{array}\right|, \\
& k=1,2, \ldots, 8 \text {. }
\end{aligned}
$$

Аналогичным образом из (32)-(34) имеем

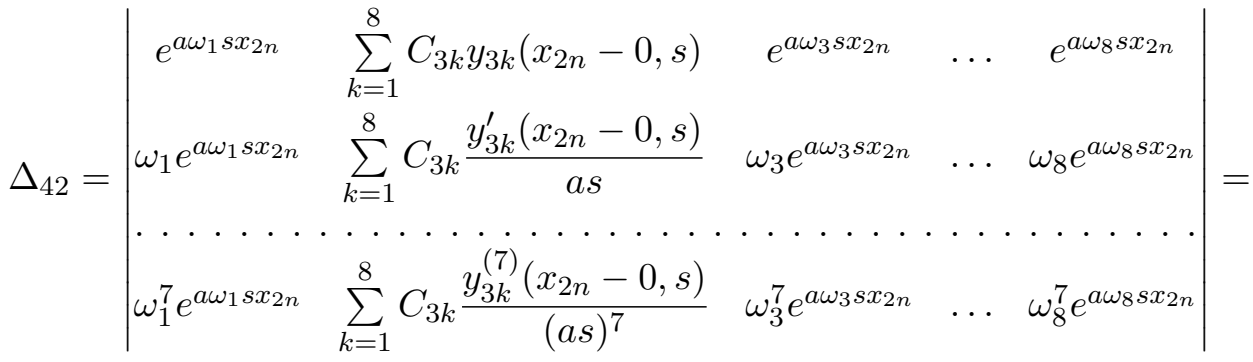

$$
\begin{aligned}
& =e^{a \omega_{1} s x_{2 n}} e^{a \omega_{3} s x_{2 n}}(\ldots) e^{a \omega_{8} s x_{2 n}} \sum_{k=1}^{8} C_{3 k} \Delta_{42 k}= \\
& =C_{31} \Delta_{421}+C_{32} \Delta_{422}+\cdots+C_{38} \Delta_{428} \text {; }
\end{aligned}
$$

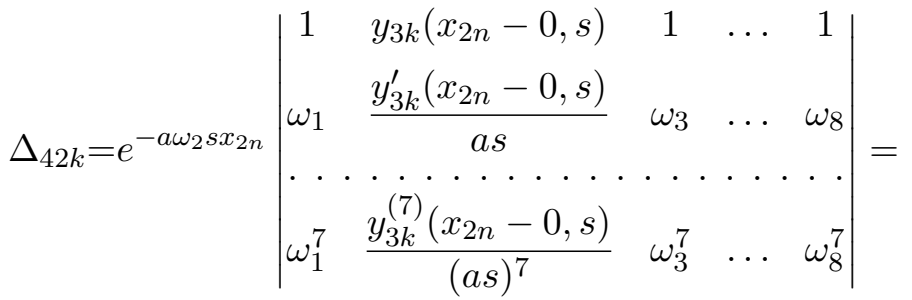

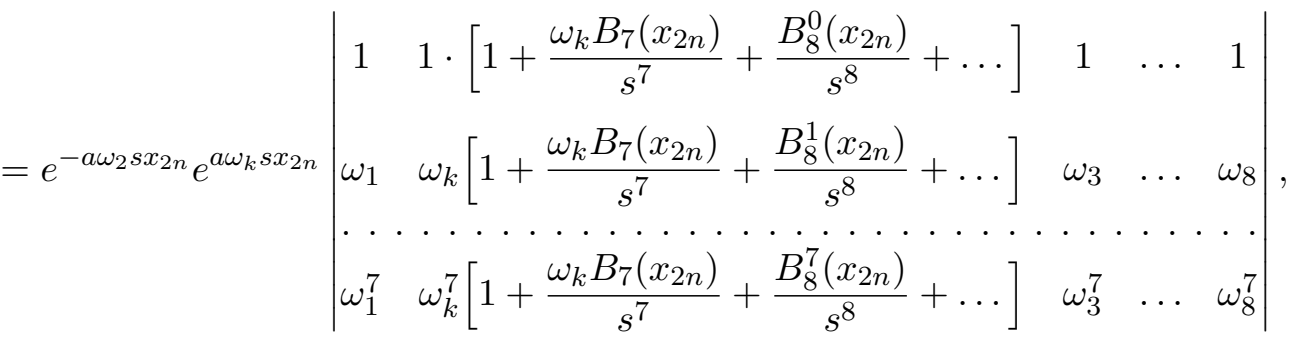

$$
\begin{aligned}
& k=1,2, \ldots, 8 \text {. }
\end{aligned}
$$


Разложим определитель $\Delta_{41 k}(k=1,2, \ldots, 8)$ из формулы (36) по первому столбцу на сумму определителей, применим формулы (30) из теоремы 5 и получим

$$
\begin{aligned}
& \Delta_{41 k}=e^{-a \omega_{1} s x_{2 n}} e^{a \omega_{k} s x_{2 n}}\left[\Delta_{41 k, 0}+\frac{\Delta_{41 k, 7}}{s^{7}}+\frac{\Delta_{41 k, 8}}{s^{8}}+\underline{O}\left(\frac{1}{s^{9}}\right)\right], \\
& k=1,2, \ldots, 8 \text {; } \\
& \Delta_{41 k, 0}=\left|\begin{array}{ccccc}
1 & 1 & 1 & \ldots & 1 \\
\omega_{k} & \omega_{2} & \omega_{3} & \ldots & \omega_{8} \\
\cdot & \ldots & \ldots & \ldots & . \\
\omega_{k}^{7} & \omega_{2}^{7} & \omega_{3}^{7} & \ldots & \omega_{8}^{7}
\end{array}\right|=\left[\begin{array}{cl}
\Delta_{00}, & k=1 ; \\
0, & k=2,3, \ldots, 8,
\end{array}\right. \\
& \Delta_{41 k, 7}=\omega_{k} B_{7}\left(x_{2 n}\right) \Delta_{41 k, 0}=\left[\begin{array}{cl}
\Delta_{00} \omega_{k} B_{7}\left(x_{2 n}\right), & k=1 ; \\
0, & k=2,3, \ldots, 8,
\end{array}\right. \\
& =\frac{\Delta_{00}}{8}\left[B_{8}^{0}\left(x_{2 n}\right) \cdot 1+B_{8}^{1}\left(x_{2 n}\right) \frac{\omega_{k}}{\omega_{1}}+B_{8}^{2}\left(x_{2 n}\right)\left(\frac{\omega_{k}}{\omega_{1}}\right)^{2}+\cdots+B_{8}^{7}\left(x_{2 n}\right)\left(\frac{\omega_{k}}{\omega_{1}}\right)^{7}\right] \text {, } \\
& k=1,2, \ldots, 8 \text {. }
\end{aligned}
$$

Подставляя в формулу (42) $k=1$, находим

$$
\Delta_{411,8}=\frac{\Delta_{00}}{8} \sum_{k=0}^{7} B_{8}^{k}\left(x_{2 n}\right) \stackrel{(26)}{=} \frac{\Delta_{00}}{8} E_{8} .
$$

Используя формулы (25), (26), из формулы (42) при $k=2,3, \ldots, 8$ получаем

$$
\begin{aligned}
& \Delta_{41 k, 8}=\frac{\Delta_{00}}{8} \frac{1}{16 a^{8}}\left[(-7) q_{3}\left(x_{0}\right) \sum_{n=0}^{7}\left(\frac{\omega_{k}}{\omega_{1}}\right)^{n}+\right. \\
& \left.\quad+q_{3}\left(x_{2 n}\right) \sum_{m=0}^{7}(7-2 m)\left(\frac{\omega_{k}}{\omega_{1}}\right)^{m}\right]=\Delta_{00} V_{k}, \\
& V_{k}=\frac{q_{3}\left(x_{2 n}\right) G_{k}}{128 a^{8}}, \quad G_{k}=\sum_{m=0}^{7}(7-2 m)\left(\frac{\omega_{k}}{\omega_{1}}\right)^{m}, \quad m=2,3, \ldots, 8, \quad
\end{aligned}
$$

так как $\sum_{n=0}^{7}\left(\frac{\omega_{k}}{\omega_{1}}\right)^{n}=0$ в силу формул $(10)-(12)$. При этом

$$
G_{5}=8=7+5 \omega_{5}+3 \omega_{5}^{2}+\omega_{5}^{3}-\omega_{5}^{4}-3 \omega_{5}^{5}-7 \omega_{5}^{7}, \quad \omega_{5} \stackrel{(10)}{=}-1=-\omega_{1} .
$$

Вычисляя аналогичным образом определители $\Delta_{42}$ и $\Delta_{42 k}$ из (37), (38), находим

$$
\begin{array}{r}
\Delta_{42 k}=e^{-a \omega_{2} s x_{2 n}} e^{a \omega_{k} s x_{2 n}}\left[\Delta_{42 k, 0}+\frac{\Delta_{42 k, 7}}{s^{7}}+\frac{\Delta_{42 k, 8}}{s^{8}}+\underline{O}\left(\frac{1}{s^{9}}\right)\right], \\
k=1,2, \ldots, 8 ;
\end{array}
$$




$$
\begin{aligned}
& \Delta_{42 k, 0}=\left|\begin{array}{ccccc}
1 & 1 & 1 & \ldots & 1 \\
\omega_{1} & \omega_{k} & \omega_{3} & \ldots & \omega_{8} \\
\hdashline & \cdots & \ldots & \ldots & \ldots \\
\omega_{1}^{7} & \omega_{k}^{7} & \omega_{3}^{7} & \ldots & \omega_{8}^{7}
\end{array}\right|=\left[\begin{array}{cc}
\Delta_{00}, & k=2 \\
0, & k=1,3,4, \ldots, 8
\end{array}\right. \\
& \Delta_{42 k, 7}=\omega_{k} B_{7}\left(x_{2 n}\right) \Delta_{42 k, 0}=\left[\begin{array}{cl}
\Delta_{00} \omega_{k} B_{7}\left(x_{2 n}\right), & k=2 ; \\
0, & k=1,3,4, \ldots, 8,
\end{array}\right. \\
& \Delta_{42 k, 8}=\sum_{n=0}^{7} B_{8}^{0}\left(x_{2 n}\right) \omega_{k}^{n} \delta_{n+1,2} \stackrel{(30)}{=} \frac{\Delta_{00}}{8} \sum_{n=0}^{7} B_{8}^{n}\left(x_{2 n}\right)\left(\frac{\omega_{k}}{\omega_{2}}\right) \stackrel{(10)}{=} \\
& =\frac{\Delta_{00}}{8} \sum_{n=0}^{7} B_{8}^{n}\left(x_{2 n}\right)\left(\frac{\omega_{k-1}}{\omega_{1}}\right)^{n}=\Delta_{00} V_{k-1}, \quad k=1,3,4, \ldots, 8, \\
& \Delta_{422,8} \stackrel{(49),(43)}{=} \frac{\Delta_{00}}{8} E_{8} \text {. }
\end{aligned}
$$

При этом $\omega_{k+8}=\omega_{k}, G_{k+8}=G_{k}, V_{k+8}=V_{k}, k=1,2, \ldots, 8$.

Вычисляя аналогичным образом определители $\Delta_{43}, \Delta_{44}, \ldots, \Delta_{48}$ из (32)(34), применяя формулы (35)-(50), приходим к выводу о справедливости следующей теоремы.

Теорема 6. Для определителей $\Delta_{4 n}(n=1,2, \ldots, 8)$ из (32)-(34) справедливы следующие формуль:

$$
\Delta_{4 n}=\sum_{k=1}^{8} C_{3 k} \Delta_{4 n k}=C_{31} \Delta_{4 n 1}+C_{32} \Delta_{4 n 2}+\cdots+C_{38} \Delta_{4 n 8} .
$$

При этом элементы матрицы

$$
\Delta_{4 n k}=\left(\begin{array}{cccc}
\Delta_{411} & \Delta_{412} & \ldots & \Delta_{418} \\
\Delta_{421} & \Delta_{422} & \ldots & \Delta_{428} \\
\ldots & \ldots & \ldots & \ldots \\
\Delta_{481} & \Delta_{482} & \ldots & \Delta_{488}
\end{array}\right), \quad n, k=1,2, \ldots, 8
$$

находятся по следующим формулам:

$$
\begin{array}{r}
\Delta_{4 k k}=e^{a\left(\omega_{k}-\omega_{k}\right) s x_{2 n}} \Delta_{00}\left[1+\frac{\omega_{k} B_{7}\left(x_{2 n}\right)}{s^{7}}+\frac{E_{8}}{8 s^{8}}+\underline{O}\left(\frac{1}{s^{9}}\right)\right], \\
k=1,2, \ldots, 8 ; \\
\Delta_{4 k m}=e^{a\left(\omega_{m}-\omega_{k}\right) s x_{2 n}} \Delta_{00}\left[0+\frac{0}{s^{7}}+\frac{V_{m-k+1}}{s^{8}}+\underline{O}\left(\frac{1}{s^{9}}\right)\right], \\
m \neq k ; \quad V_{m \pm 8}=V_{m} ; \quad k, m=1,2, \ldots, 8,
\end{array}
$$

где числа $V_{k}$ определены формулой (44). 
4. Изучение условий склейки (7). Применяя формулы (21) и (16), из условий склейки (7) получаем

$$
\left\{\begin{array}{c}
y_{3}\left(x_{0}+0, s\right) \stackrel{(7)}{=} y_{2}\left(x_{0}-0, s\right) \Leftrightarrow \sum_{k=1}^{8} C_{3 k} y_{3 k}\left(x_{0}+0, s\right)= \\
\quad=\sum_{k=1}^{8} C_{2 k} y_{2 k}\left(x_{0}-0, s\right) ; \\
\frac{y_{3}^{(m)}\left(x_{0}+0, s\right)}{(a s)^{m}} \stackrel{(7)}{=} \frac{y_{2}^{(m)}\left(x_{0}-0, s\right)}{(a s)^{m}} \Leftrightarrow \sum_{k=1}^{8} C_{3 k} \frac{y_{3 k}^{(m)}\left(x_{0}+0, s\right)}{(a s)^{m}}= \\
=\sum_{k=1}^{8} C_{2 k} \frac{y_{2 k}^{(m)}\left(x_{0}-0, s\right)}{(a s)^{m}}, \quad m=1,2, \ldots, 7 .
\end{array}\right.
$$

Из метода Крамера и общей теории свойств решений дифференциального уравнения (3) следует, что система (54) имеет единственное решение, которое представляется в виде

$$
\begin{gathered}
C_{31}=\frac{\Delta_{31}}{\Delta_{03}(s) \neq 0}, \quad C_{32}=\frac{\Delta_{32}}{\Delta_{03}(s)}, \quad \ldots, \quad C_{38}=\frac{\Delta_{38}}{\Delta_{03}(s)} ; \\
\left.\Delta_{03}(s)=\Delta_{03}(x, s)=\mid \begin{array}{llll}
y_{31}(x, s) & y_{32}(x, s) & \ldots & y_{38}(x, s) \\
\frac{y_{31}^{\prime}(x, s)}{a s} & \frac{y_{32}^{\prime}(x, s)}{a s} & \ldots & \frac{y_{38}^{\prime}(x, s)}{a s} \\
\ldots \ldots \ldots & \ldots \ldots
\end{array}\right]_{x=x_{0} \pm 0}^{(7)} \ldots . . .
\end{gathered}
$$

Так как $\Delta_{03}(x, s)$ - определитель Вронского фундаментальной системы решений $\left\{y_{3 k}(x, s)\right\}_{k=1}^{8}$ дифференциального уравнения (3), он не зависит от $x$ и не равен нулю. Определители $\Delta_{3 k}(s)(k=1,2, \ldots, 8)$ получаются из определителя $\Delta_{03}(x, s)$ из $(56)$ заменой $k$-го столбца на столбец

$$
\left(\sum_{k=1}^{8} C_{2 k} y_{2 k}\left(x_{0}-0, s\right) \sum_{k=1}^{8} C_{2 k} \frac{y_{2 k}^{\prime}\left(x_{0}-0, s\right)}{a s} \ldots \sum_{k=1}^{8} C_{2 k} \frac{y_{2 k}^{(7)}\left(x_{0}-0, s\right)}{(a s)^{7}}\right)^{\top} .
$$

Применяя формулы $(22)-(26)$, определитель $\Delta_{03}(s)$ из (56) можно представить в виде

$$
\begin{aligned}
& \Delta_{03}(s)=
\end{aligned}
$$

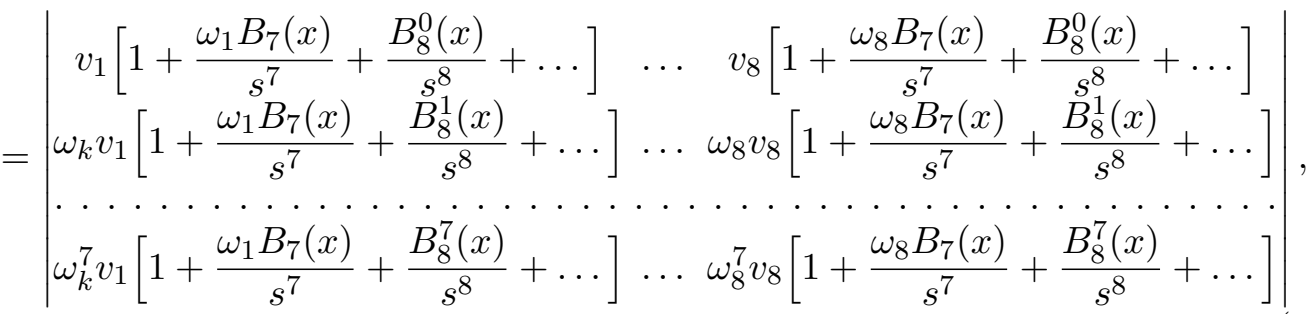


где

$$
\begin{gathered}
v_{k}=e^{a \omega_{k} s x}, \quad k=1,2, \ldots, 8 \\
v_{1} v_{2}(\ldots) v_{8}=e^{a\left(\omega_{1}+\omega_{2}+\cdots+\omega_{8}\right) s x}=e^{0}=1 .
\end{gathered}
$$

Для вычисления определителя $\Delta_{03}(s)$ из (58) вынесем из $k$-го столбца множитель $v_{k}=e^{a \omega_{k} s x}$, затем разложим по столбцам на сумму определителей, получим

$$
\begin{gathered}
\Delta_{03}(s)=\Delta_{00}+\frac{\Delta_{03,7}(s)}{s^{7}}+\frac{\Delta_{03,8}(s)}{s^{8}}+\underline{O}\left(\frac{1}{s^{9}}\right) \\
\Delta_{03,7}(s) \stackrel{(29)}{=} \omega_{1} B_{7}(x) \Delta_{00}+\omega_{2} B_{7}(x) \Delta_{00}+\cdots+\omega_{8} B_{7}(x) \Delta_{00}= \\
=\Delta_{00} B_{7}(x) \sum_{k=1}^{8} \omega_{k} \stackrel{(12)}{=} 0 \\
\Delta_{03,8}(s)=B_{8}^{0}(x) \sum_{k=1}^{8}(-1)^{k-1} \cdot 1 \cdot \delta_{1 k}+B_{8}^{1}(x) \sum_{k=1}^{8}(-1)^{k} \omega_{k} \delta_{2 k}+ \\
+B_{8}^{2}(x) \sum_{k=1}^{8}(-1)^{k-1} \omega_{k}^{2} \delta_{3 k}+\cdots+B_{8}^{7}(x) \sum_{k=1}^{8}(-1)^{k} \omega_{k}^{7} \delta_{8 k} \stackrel{(28)}{=} \\
=\Delta_{00} \sum_{m=0}^{7} B_{8}^{m}(x) \stackrel{(26)}{=} \Delta_{00} E_{8} .
\end{gathered}
$$

Поэтому из формул (59)-(61) следует формула

$$
\Delta_{03}(s)=\Delta_{00}\left[1+\frac{0}{s^{7}}+\frac{E_{8}}{s^{8}}+\underline{O}\left(\frac{1}{s^{9}}\right)\right] \neq 0, \quad E_{8} \stackrel{(26)}{=} \frac{-7 q_{3}\left(x_{0}\right)}{2 a^{8}} .
$$

Из формул (56), (57) следует формула для вычисления определителя $\Delta_{31}$ из (55):

$$
\begin{aligned}
& \Delta_{31}=\left|\begin{array}{rrrr}
\sum_{k=1}^{8} C_{2 k} y_{2 k}\left(x_{0}-0, s\right) & y_{32}\left(x_{0}+0, s\right) & \ldots & y_{38}\left(x_{0}+0, s\right) \\
\sum_{k=1}^{8} C_{2 k} \frac{y_{2 k}^{\prime}\left(x_{0}-0, s\right)}{a s} & \frac{y_{32}^{\prime}\left(x_{0}+0, s\right)}{a s} & \ldots & \frac{y_{38}^{\prime}\left(x_{0}+0, s\right)}{a s} \\
\ldots \ldots \ldots \ldots \ldots \ldots & \ldots \ldots \ldots & \ldots \ldots \\
\sum_{k=1}^{8} C_{2 k} \frac{y_{2 k}^{(7)}\left(x_{0}-0, s\right)}{(a s)^{7}} & \frac{y_{32}^{(7)}\left(x_{0}+0, s\right)}{(a s)^{7}} & \ldots & \frac{y_{38}\left(x_{0}+0, s\right)}{(a s)^{7}}
\end{array}\right|= \\
&=\sum_{k=1}^{8} C_{2 k} \Delta_{31 k}=C_{21} \Delta_{311}+C_{22} \Delta_{312}+\ldots+C_{28} \Delta_{318} .
\end{aligned}
$$

При этом, применяя формулы (58) и (16), (17), имеем 


$$
\begin{aligned}
& \Delta_{31 k}=
\end{aligned}
$$

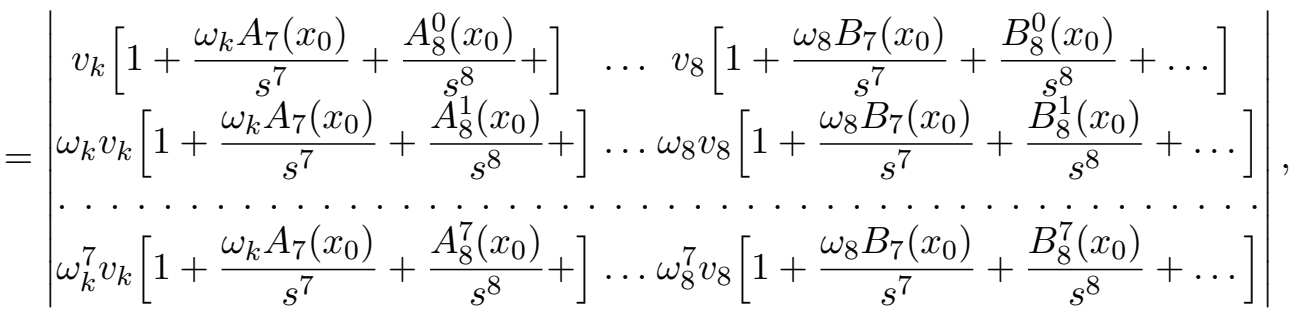

$$
\begin{aligned}
& v_{k}=e^{a \omega_{k} s x_{0}}, \quad k=1,2, \ldots, 8 ; \quad B_{7}\left(x_{0}\right) \stackrel{(24)}{=} 0 .
\end{aligned}
$$

Вычисляя определители $\Delta_{31 k}(k=1,2, \ldots, 8)$ из (64) аналогично вычислению определителей $\Delta_{41 k}(k=1,2, \ldots, 8)$ из $(36),(39)-(45)$, получаем

$$
\begin{array}{r}
\Delta_{31 k}=e^{a \omega_{k} s x_{0}} e^{-a \omega_{1} s x_{0}}\left[\Delta_{31 k, 0}+\frac{\Delta_{31 k, 7}}{s^{7}}+\frac{\Delta_{31 k, 8}}{s^{8}}+\underline{O}\left(\frac{1}{s^{9}}\right)\right], \\
k=1,2, \ldots, 8,
\end{array}
$$

$\Delta_{31 k, 0}=\left[\begin{array}{cl}\Delta_{00}, & k=1 ; \\ 0, & k=2,3, \ldots, 8,\end{array} \Delta_{31 k, 7}=\left[\begin{array}{cl}\Delta_{00} \omega_{1} A_{7}\left(x_{0}\right), & k=1 ; \\ 0, & k=2,3, \ldots, 8,\end{array}\right.\right.$

$$
\begin{gathered}
\Delta_{311,8}=e^{a \omega_{1} s x_{0}} e^{-a \omega_{1} s x_{0}}\left[\sum_{m=0}^{7} A_{8}^{m}\left(x_{0}\right)(-1)^{m} \omega_{1}^{m} \delta_{m+1,1}+\right. \\
\left.+\sum_{m=0}^{7} B_{8}^{m}\left(x_{0}\right)\left(\sum_{n=2}^{8}(-1)^{m+n-1} \omega_{n}^{m} \delta_{m+1, n}\right)\right]=\frac{\Delta_{00}}{8}\left[D_{8}+7 E_{8}\right], \\
D_{8} \stackrel{(20)}{=} \frac{-7 q_{2}\left(x_{1 n}\right)}{2 a^{8}}
\end{gathered}
$$

$$
\begin{gathered}
\Delta_{31 k, 8}=\frac{\Delta_{00}}{8} \sum_{n=0}^{7}\left[A_{7}^{n}\left(x_{0}\right)-B_{7}^{n}\left(x_{0}\right)\right]\left(\frac{\omega_{k}}{\omega_{1}}\right)^{n}=\Delta_{00} R_{k}, \\
R_{k}=\frac{1}{128 a^{8}} \Delta \widetilde{q}\left(x_{0}\right) G_{k} ; \quad \Delta \widetilde{q}\left(x_{0}\right)=q_{2}\left(x_{0}-0\right)-q_{3}\left(x_{0}+0\right), \\
k=2,3, \ldots, 8,
\end{gathered}
$$

где числа $G_{k}$ определены формулой (44).

Определители $\Delta_{32}, \Delta_{33}, \ldots, \Delta_{38}$ из (55)-(57) выписываются и вычисляются аналогично определителям $\Delta_{4 k}(k=2,3, \ldots, 8)$ из $(37),(38),(46)-(53)$, в результате придем к выводу о справедливости следующей теоремы.

Теорема 7. Для определителей $\Delta_{3 n}(n=1,2, \ldots, 8)$ из (55)-(57) справедливы следующие формулы:

$$
\begin{array}{r}
\Delta_{3 n}=\sum_{k=1}^{8} C_{2 k} \Delta_{3 n k}=C_{21} \Delta_{3 n 1}+C_{22} \Delta_{3 n 2}+\cdots+C_{28} \Delta_{3 n 8} \\
n=1,2, \ldots, 8 .
\end{array}
$$


При этом элементы матрищы

$$
\Delta_{3 n k}=\left(\begin{array}{cccc}
\Delta_{311} & \Delta_{312} & \ldots & \Delta_{318} \\
\Delta_{321} & \Delta_{322} & \ldots & \Delta_{328} \\
\ldots & \ldots & \ldots & \ldots \\
\Delta_{381} & \Delta_{382} & \ldots & \Delta_{388}
\end{array}\right), \quad n, k=1,2, \ldots, 8
$$

находятся по формулам

$$
\begin{array}{r}
\Delta_{3 k k}=\Delta_{00} e^{a\left(\omega_{k}-\omega_{k}\right) s x_{0}}\left[1+\frac{\omega_{k} A_{7}\left(x_{0}\right)}{s^{7}}+\frac{H_{8}}{s^{8}}+\underline{O}\left(\frac{1}{s^{9}}\right)\right], \\
H_{8}=\frac{D_{8}+7 E_{8}}{8}, \quad k=1,2, \ldots, 8 ; \\
\Delta_{3 k m}=\Delta_{00} e^{a\left(\omega_{m}-\omega_{k}\right) s x_{0}}\left[0+\frac{0}{s^{7}}+\frac{R_{m-k+1}}{s^{8}}+\underline{O}\left(\frac{1}{s^{9}}\right)\right], \\
m \neq k ; \quad R_{m \pm 8}=R_{8}, \quad m, k=1,2, \ldots, 8
\end{array}
$$

где величины $R_{k}$ определены формулой (68).

Доказательство формул (69)-(71) проводится обобщением формул (63)(68).

5. Изучение условий склейки (6). Применяя формулы (13) и (15), из условий склейки (6) получаем

$$
\left\{\begin{array}{c}
y_{2}\left(x_{1 n}+0, s\right) \stackrel{(6)}{=} y_{1}\left(x_{1 n}-0, s\right) \Leftrightarrow \sum_{k=1}^{8} C_{2 k} y_{2 k}\left(x_{1 n}+0, s\right)= \\
=\sum_{k=1}^{8} C_{1 k} y_{1 k}\left(x_{1 n}-0, s\right) ; \\
\frac{y_{2}^{(m)}\left(x_{1 n}+0, s\right)}{(a s)^{m}} \stackrel{(6)}{=} \frac{y_{1}^{(m)}\left(x_{1 n}-0, s\right)}{(a s)^{m}} \Leftrightarrow \sum_{k=1}^{8} C_{2 k} \frac{y_{2 k}^{(m)}\left(x_{1 n}+0, s\right)}{(a s)^{m}}= \\
=\sum_{k=1}^{8} C_{1 k} \frac{y_{1 k}^{(m)}\left(x_{1 n}-0, s\right)}{(a s)^{m}}, \quad m=1,2, \ldots, 7 .
\end{array}\right.
$$

Решая систему (72) методом Крамера, аналогично решению систем (31) и (54), получаем

$$
\begin{aligned}
& C_{21}=\frac{\Delta_{21}}{\Delta_{02}(s) \neq 0}, \quad C_{22}=\frac{\Delta_{22}}{\Delta_{02}(s)}, \quad \ldots, \quad C_{28}=\frac{\Delta_{28}}{\Delta_{02}(s)} ;
\end{aligned}
$$

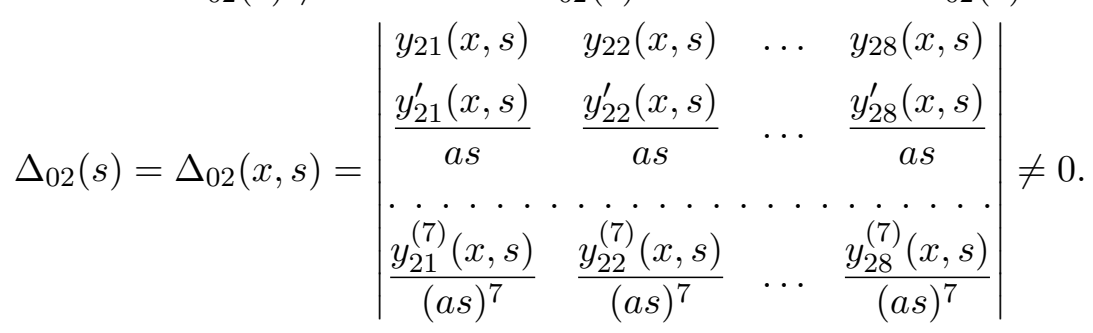


Определитель $\Delta_{02}(x, s)$ - определитель Вронского фундаментальной системы решений $\left\{y_{2 k}(x, s)\right\}_{k=1}^{8}$ дифференциального уравнения $(2)$, поэтому он не зависит от $x$ и не равен нулю. Определители $\Delta_{2 k}(k=1,2, \ldots, 8)$ получаются из определителя $\Delta_{02}(x, s)$ из $(74)$ заменой $k$-го столбца на столбец

$$
\left(\sum_{k=1}^{8} C_{1 k} y_{1 k}\left(x_{1 n}-0, s\right) \sum_{k=1}^{8} C_{1 k} \frac{y_{1 k}^{\prime}\left(x_{1 n}-0, s\right)}{a s} \ldots \sum_{k=1}^{8} C_{1 k} \frac{y_{1 k}^{(7)}\left(x_{1 n}-0, s\right)}{(a s)^{7}}\right)^{\top} .
$$

Применяя формулы (16)-(20) и (14), аналогично выводу формул (58)-(71) можно доказать следующее утверждение.

Теорема 8. Для определителей $\Delta_{2 n}(n=1,2, \ldots, 8)$ из (73)-(75) справедливы следующие бормуль:

$$
\Delta_{2 n}=\sum_{k=1}^{8} C_{1 k} \Delta_{2 n k}=C_{11} \Delta_{2 n 1}+C_{12} \Delta_{2 n 2}+\cdots+C_{18} \Delta_{2 n 8}, \quad n=1,2, \ldots, 8,
$$

при этом элементы матрицы

$$
\Delta_{2 n k}=\left(\begin{array}{cccc}
\Delta_{211} & \Delta_{212} & \ldots & \Delta_{218} \\
\Delta_{221} & \Delta_{222} & \ldots & \Delta_{228} \\
\ldots & \ldots & \ldots & \ldots \\
\Delta_{281} & \Delta_{282} & \ldots & \Delta_{288}
\end{array}\right), \quad n, k=1,2, \ldots, 8
$$

вычисляются по формулам

$$
\begin{gathered}
\Delta_{2 k k}=\Delta_{00} e^{a\left(\omega_{k}-\omega_{k}\right) s x_{1 n}}\left[1+\frac{0}{s^{7}}+\frac{7 D_{8}}{s^{8}}+\underline{O}\left(\frac{1}{s^{9}}\right)\right], \quad k=1,2, \ldots, 8, \\
\Delta_{2 k m}=\Delta_{00} e^{a\left(\omega_{m}-\omega_{k}\right) s x_{1 n}}\left[0+\frac{0}{s^{7}}+\frac{T_{m-k+1}}{s^{8}}+\underline{O}\left(\frac{1}{s^{9}}\right)\right], \quad m \neq k ; \\
T_{k}=\frac{-q_{2}\left(x_{1 n}\right) G_{k}}{128 a^{8}}, \quad T_{k \pm 8}=T_{k} ; \quad m, k=1,2, \ldots, 8
\end{gathered}
$$

где величины $G_{k}$ определены бормулами (44), (45).

6. Изучение граничных условий (9). Из первых шести граничных условий (9) с помощью формул (13), (14) получаем

$$
\begin{aligned}
\frac{y_{1}^{\left(m_{p}\right)}(0, s)}{(a s)^{m_{p}}} \stackrel{(9)}{=} 0 & \Leftrightarrow \sum_{k=1}^{8} C_{1 k} \frac{y_{1 k}^{\left(m_{p}\right)}(0, s)}{(a s)^{m_{p}}}=0 \Leftrightarrow \\
\Leftrightarrow \quad \sum_{k=1}^{8} C_{1 k} \omega_{k}^{m_{p}} e^{0}=0 & \Leftrightarrow \sum_{k=1}^{8} C_{1 k} \omega_{k}^{m_{p}}=0, \quad p=1,2, \ldots, 6 .
\end{aligned}
$$

Из последних двух граничных условий (9), применяя формулы (27), (28), получаем

$$
\frac{y_{4}^{\left(n_{p}\right)}(\pi, s)}{(a s)^{n_{p}}} \stackrel{(9)}{=} 0 \Leftrightarrow \sum_{k=1}^{8} C_{4 k} \frac{y_{4 k}^{\left(n_{p}\right)}(\pi, s)}{(a s)^{n_{p}}}=0 \Leftrightarrow
$$




$$
\Leftrightarrow \quad \sum_{k=1}^{8} C_{4 k} \omega_{k}^{n_{p}} e^{a \omega_{k} s \pi}=0, \quad p=1,2
$$

Применяя формулы (32) и (51)-(53), из (79) выводим, что

$$
\begin{gathered}
\frac{y_{4}^{\left(n_{p}\right)}(\pi, s)}{(a s)^{n_{p}}}=0 \Leftrightarrow \sum_{k=1}^{8} \frac{\Delta_{4 k}}{\Delta_{04}(s)} \omega_{k}^{n_{p}} e^{a \omega_{k} s \pi}=0 \Leftrightarrow \\
\Leftrightarrow \frac{1}{\Delta_{04}(s)} \sum_{k=1}^{8}\left(\sum_{n=1}^{8} C_{3 n} \Delta_{4 k n}\right) \omega_{k}^{n_{p}} e^{a \omega_{k} s \pi}=0 \Leftrightarrow \\
\Leftrightarrow \quad \sum_{k=1}^{8} C_{3 k} \psi_{3 k, n_{p}}(\pi, s)=0, \quad p=1,2,
\end{gathered}
$$

где $\Delta_{04}(s) \neq 0$ и введены обозначения

$$
\psi_{3 k, n_{p}}(\pi, s)=\sum_{n=1}^{8} \Delta_{4 k n} \omega_{n}^{n_{p}} e^{a \omega_{n} s \pi}, \quad k=1,2, \ldots, 8 ; \quad p=1,2 .
$$

Подставляя формулы (55) и (69)-(71) в формулу (80), получаем

$$
\begin{gathered}
\frac{y_{4}^{\left(n_{p}\right)}(\pi, s)}{(a s)^{n_{p}}}=0 \Leftrightarrow \sum_{k=1}^{8} \frac{\Delta_{3 k}}{\Delta_{03}(s)} \psi_{3 k, n_{p}}(\pi, s)=0, \quad \Delta_{03}(s) \neq 0 \Leftrightarrow \\
\Leftrightarrow \frac{1}{\Delta_{03}(s)} \sum_{k=1}^{8}\left(\sum_{n=1}^{8} C_{2 n} \Delta_{3 k n}\right) \psi_{3 k, n_{p}}(\pi, s)=0 \Leftrightarrow \\
\Leftrightarrow \sum_{k=1}^{8} C_{2 k} \phi_{2 k, n_{p}}(\pi, s)=0, \\
\phi_{2 k, n_{p}}(\pi, s)=\sum_{n=1}^{8} \Delta_{3 k n} \psi_{3 n, n_{p}}(\pi, s), \quad k=1,2, \ldots, 8 ; \quad p=1,2 .
\end{gathered}
$$

Применяя формулы (73) и (76), (77), из (82) находим

$$
\begin{gathered}
\frac{y_{4}^{\left(n_{p}\right)}(\pi, s)}{(a s)^{n_{p}}}=0 \Leftrightarrow \sum_{k=1}^{8} \frac{\Delta_{2 k}}{\Delta_{02}(s)} \phi_{2 k, n_{p}}(\pi, s)=0, \quad \Delta_{02}(s) \neq 0 \Leftrightarrow \\
\Leftrightarrow \frac{1}{\Delta_{02}(s)} \sum_{k=1}^{8}\left(\sum_{n=1}^{8} C_{1 n} \Delta_{2 k n}\right) \phi_{2 k, n_{p}}(\pi, s)=0 \Leftrightarrow \\
\Leftrightarrow \sum_{k=1}^{8} C_{1 k} u_{1 k, n_{p}}(\pi, s)=0,
\end{gathered}
$$

$u_{1 k, n_{p}}(\pi, s)=\sum_{n=1}^{8} \Delta_{2 k n} \phi_{2 n, n_{p}}(\pi, s), \quad k=1,2, \ldots, 8 ; \quad p=1,2$. 
Объединяя (78) и (84) в одну систему, получим систему из восьми однородных уравнений с восемью неизвестными $C_{11}, C_{12}, \ldots, C_{18}$, которая имеет ненулевые решения только в том случае, когда ее определитель равен нулю. Поэтому справедливо следующее утверждение.

Теорема 9. Уравнение на собственные значения дифференииалъного оператора (1)-(8) с граничными условиями (9) имеет следующий вид:

$$
\begin{aligned}
& f(s)=
\end{aligned}
$$

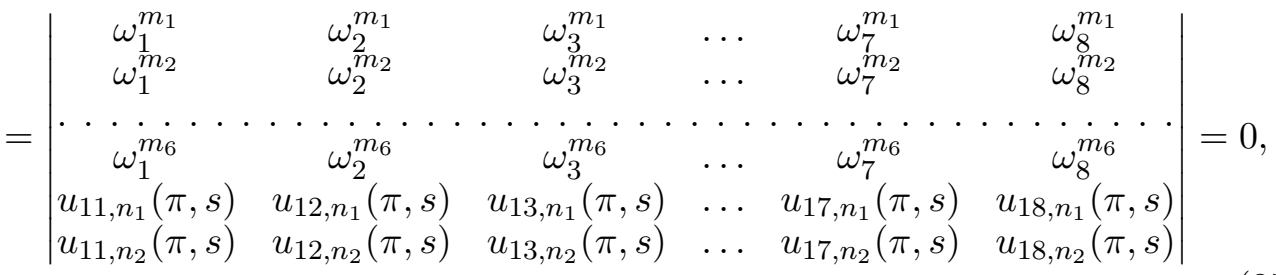

$$
u_{1 k, n_{p}}(\pi, s)=\sum_{n=1}^{8} \Delta_{2 k n} \phi_{2 n, n_{p}}(\pi, s) \stackrel{(83)}{=} \sum_{n=1}^{8} \Delta_{2 n k}\left(\sum_{m=1}^{8} \Delta_{3 m n} \phi_{3 m, n_{p}}(\pi, s)\right) .
$$

Применяя формулы (70), (71) и (78), (77), оставляя только главные по росту $s$ величины, проводя оценки величин с точностью до $\underline{O}\left(s^{-8}\right)$, в формуле (86) имеем

$$
\begin{aligned}
& u_{1 k, n_{p}}(\pi, s)=\Delta_{2 k k} \Delta_{3 k k} \psi_{3 k, n_{p}}+\Delta_{2 k k} \sum_{\substack{m=1 \\
m \neq k}}^{8} \Delta_{3 m k} \psi_{3 m, n_{p}}(\pi, s)+ \\
& \quad+\Delta_{3 k k} \sum_{\substack{m=1 \\
m \neq k}}^{8} \Delta_{2 m k} \psi_{3 m, n_{p}}(\pi, s)+\underline{O}\left(\frac{1}{s^{16}}\right), \quad k=1,2, \ldots, 8 ; p=1,2 .
\end{aligned}
$$

Подставляя в формулу (87) формулу для $\psi_{3 m, n_{p}}(\pi, s)$ из $(81)$ и оставляя только главные по росту $s$ величины, получаем

$$
\begin{aligned}
& u_{1 k, n_{p}}(\pi, s)=\Delta_{2 k k} \Delta_{3 k k} \Delta_{4 k k} \omega_{k}^{n_{p}} e^{a \omega_{k} s \pi}+ \\
& +\Delta_{2 k k} \Delta_{3 k k} \sum_{\substack{m=1 \\
m \neq k}}^{8} \Delta_{4 m k} \omega_{m}^{n_{p}} e^{a \omega_{m} s \pi}+\Delta_{2 k k} \Delta_{4 k k} \sum_{m=1}^{8} \Delta_{3 m k} \omega_{m}^{n_{p}} e^{a \omega_{m} s \pi}+ \\
& +\Delta_{3 k k} \Delta_{4 k k} \sum_{m=1}^{8} \Delta_{2 m k} \omega_{m}^{n_{p}} e^{a \omega_{m} s \pi}+\underline{O}\left(\frac{1}{s^{16}}\right), k=1,2, \ldots, 8 ; \quad p=1,2 .
\end{aligned}
$$

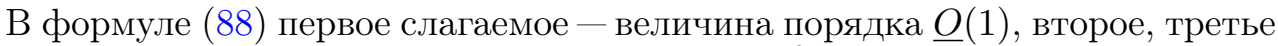
и четвертое слагаемое - величины порядка $\underline{O}\left(s^{-8}\right)$.

Применяя теорему Лапласа, разложим определитель $f(s)$ из (85) по последним двум строкам и получим 


$$
\begin{aligned}
& f(s)=\left|\begin{array}{ll}
u_{11, n_{1}}(\pi, s) & u_{12, n_{1}}(\pi, s) \\
u_{11, n_{2}}(\pi, s) & u_{12, n_{2}}(\pi, s)
\end{array}\right| D_{345678}+\left|\begin{array}{ll}
u_{12, n_{1}}(\pi, s) & u_{13, n_{1}}(\pi, s) \\
u_{12, n_{2}}(\pi, s) & u_{13, n_{2}}(\pi, s)
\end{array}\right| D_{145678}+ \\
& +\left|\begin{array}{ll}
u_{13, n_{1}}(\pi, s) & u_{14, n_{1}}(\pi, s) \\
u_{13, n_{2}}(\pi, s) & u_{14, n_{2}}(\pi, s)
\end{array}\right| D_{125678}+\cdots- \\
& -\left|\begin{array}{ll}
u_{11, n_{1}}(\pi, s) & u_{13, n_{1}}(\pi, s) \\
u_{11, n_{2}}(\pi, s) & u_{13, n_{2}}(\pi, s)
\end{array}\right| D_{245678}+\cdots=0, \\
& D_{k_{1} k_{2} k_{3} k_{4} k_{5} k_{6}}=\left|\begin{array}{cccc}
\omega_{k_{1}}^{m_{1}} & \omega_{k_{2}}^{m_{1}} & \ldots & \omega_{k_{6}}^{m_{1}} \\
\omega_{k_{1}}^{m_{2}} & \omega_{k_{2}}^{m_{2}} & \ldots & \omega_{k_{6}}^{m_{2}} \\
\dot{.} \cdot & . \cdot & \ldots & . \\
\omega_{k_{1}}^{m_{6}} & \omega_{k_{2}}^{m_{6}} & \ldots & \omega_{k_{6}}^{m_{6}}
\end{array}\right|
\end{aligned}
$$

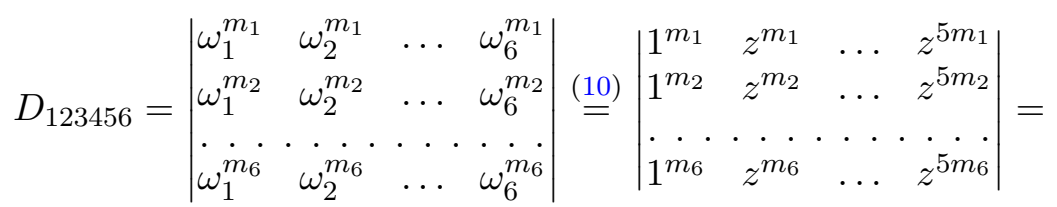$$
=\operatorname{det} \text { Wandermond's }\left(z^{m_{1}}, z^{m_{2}}, \ldots, z^{m_{6}}\right)=
$$$$
=\prod_{\substack{k>n, k, n=1,2, \ldots, 8}}\left(z^{m_{k}}-z^{m_{n}}\right)=D_{6} \neq 0 ;
$$ 


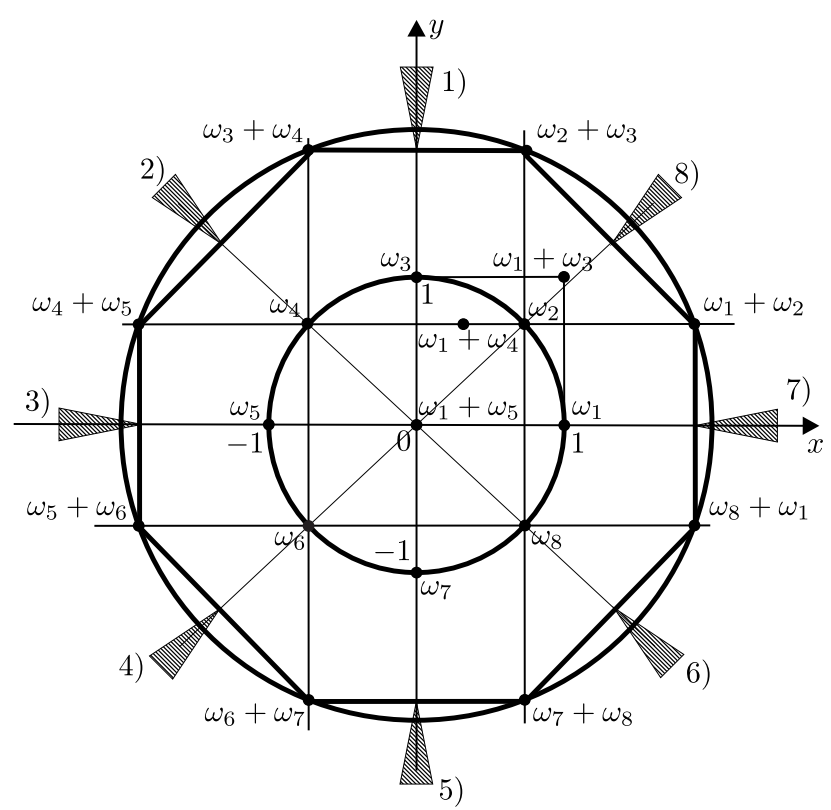

Индикаторная диаграмма уравнения (92)

[The indicator diagram for Eq. (92)]

Индикаторная диаграмма уравнения (92) имеет следующий вид (см. рисунок). Индикаторная диаграмма показывает, что на асимптотику корней уравнения (92) влияют экспоненты с показателями $\omega_{1}+\omega_{2}, \omega_{2}+\omega_{3}, \ldots, \omega_{7}+\omega_{8}$, $\omega_{8}+\omega_{1} ;$ экспоненты с показателями $\omega_{1}+\omega_{3}, \omega_{1}+\omega_{4}, \ldots, \omega_{m}+\omega_{n}$ при $|m-n| \geqslant 2$ на асимптотику корней уравнения (92) не влияют (они представляют собой бесконечно малые величины, их можно отбросить).

7. Асимптотика собственных значений в секторе 1) индикаторной диаграммы. Чтобы найти асимптотику корней уравнения (92) в секторе 1$)$, необходимо оставить экспоненты с показателями $\omega_{2}+\omega_{3}$ и $\omega_{3}+\omega_{4}$. Справедлива следующая теорема.

Теорема 10. Уравнение на собственные значения дифференииального оператора (1)-(8) с граничными условиями (9) в секторе 1) индикаторной диаграммы имеет вид

$$
g_{1}(s)=\left|\begin{array}{ll}
u_{12, n_{1}}(\pi, s) & u_{13, n_{1}}(\pi, s) \\
u_{12, n_{2}}(\pi, s) & u_{13, n_{2}}(\pi, s)
\end{array}\right|+z^{M_{6}}\left|\begin{array}{ll}
u_{13, n_{1}}(\pi, s) & u_{14, n_{1}}(\pi, s) \\
u_{13, n_{2}}(\pi, s) & u_{14, n_{2}}(\pi, s)
\end{array}\right|=0
$$

величины $u_{1 k, n_{p}}(\pi, s)$ определены формулой (88).

Поделим (93) на $z^{M_{6}} \neq 0$ и, используя свойства определителей, перепишем уравнение (93) в виде

$$
g_{1}(s)=\left|\begin{array}{ll}
u_{13, n_{1}}(\pi, s) & z^{M_{6}} u_{14, n_{1}}(\pi, s)+u_{12, n_{1}}(\pi, s) \\
u_{13, n_{2}}(\pi, s) & z^{M_{6}} u_{14, n_{2}}(\pi, s)+u_{12, n_{2}}(\pi, s)
\end{array}\right|=0
$$

причем, оставляя главные по росту $s$ для сектора 1) величины, из (88) и ри- 
сунка имеем

$$
\begin{array}{r}
u_{1 k, n_{1}}(\pi, s)=\Delta_{2 k k} \Delta_{3 k k} \Delta_{4 k k} \omega_{k}^{n_{p}} e^{a \omega_{k} s \pi}+\frac{\phi_{1 k, n_{p}}(\pi, s)}{s^{8}}+\underline{O}\left(\frac{1}{s^{9}}\right), \\
k=2,3,4 ;
\end{array}
$$

$\phi_{13, n_{p}}(\pi, s)=\omega_{2}^{n_{p}} e^{a \omega_{2} s \pi}\left[\Delta_{233} \Delta_{333} \Delta_{423}+\Delta_{233} \Delta_{422} \Delta_{323}+\Delta_{322} \Delta_{422} \Delta_{223}\right]+$ $+\omega_{4}^{n_{p}} e^{a \omega_{4} s \pi}\left[\Delta_{233} \Delta_{333} \Delta_{443}+\Delta_{233} \Delta_{444} \Delta_{343}+\Delta_{344} \Delta_{444} \Delta_{243}\right]$, $\phi_{14, n_{p}}(\pi, s)=\omega_{3}^{n_{p}} e^{a \omega_{3} s \pi}\left[\Delta_{244} \Delta_{344} \Delta_{434}+\Delta_{244} \Delta_{433} \Delta_{334}+\Delta_{333} \Delta_{433} \Delta_{234}\right]+$ $+\omega_{2}^{n_{p}} e^{a \omega_{2} s \pi}\left[\Delta_{244} \Delta_{344} \Delta_{424}+\Delta_{244} \Delta_{422} \Delta_{324}+\Delta_{322} \Delta_{422} \Delta_{224}\right]$, $\phi_{12, n_{p}}(\pi, s)=\omega_{3}^{n_{p}} e^{a \omega_{3} s \pi}\left[\Delta_{222} \Delta_{322} \Delta_{432}+\Delta_{222} \Delta_{433} \Delta_{332}+\Delta_{333} \Delta_{433} \Delta_{232}\right]+$ $+\omega_{4}^{n_{p}} e^{a \omega_{4} s \pi}\left[\Delta_{222} \Delta_{322} \Delta_{442}+\Delta_{222} \Delta_{444} \Delta_{342}+\Delta_{344} \Delta_{444} \Delta_{242}\right]$, $p=1,2$,

при этом из формул (76), (70) и (52) получаем

$$
\begin{array}{r}
\Delta_{2 k k} \Delta_{3 k k} \Delta_{4 k k}=\left[1+\frac{7 D_{8}}{8 s^{8}}+\underline{O}\left(\frac{1}{s^{9}}\right)\right]\left[1+\frac{\omega_{k} A_{7}\left(x_{0}\right)}{s^{7}}+\frac{H_{8}}{s^{8}}+\underline{O}\left(\frac{1}{s^{9}}\right)\right] \times \\
\times\left[1+\frac{\omega_{k} B_{7}\left(x_{2 n}\right)}{s^{7}}+\frac{E_{8}}{8 s^{8}}+\underline{O}\left(\frac{1}{s^{9}}\right)\right]=1+\frac{\omega_{k} P_{7}}{s^{8}}+\frac{P_{8}}{s^{8}}+\underline{O}\left(\frac{1}{s^{9}}\right)
\end{array}
$$

$$
P_{7}=A_{7}\left(x_{0}\right)+B_{7}\left(x_{2 n}\right) ; \quad P_{8}=\frac{7 D_{8}}{8}+H_{8}+\frac{E_{8}}{8}=D_{8}+E_{8}
$$

$H_{8} \stackrel{(70)}{=} \frac{D_{8}+7 E_{8}}{8} ; \quad D_{8} \stackrel{(20)}{=} \frac{-7 q_{2}\left(x_{1 n}\right)}{2 a^{8}} ; \quad E_{8} \stackrel{(26)}{=} \frac{-7 q_{3}\left(x_{0}\right)}{2 a^{8}} ; \quad k=1,2, \ldots, 8$.

С помощью формул (95)-(97) уравнение (94) запишем в виде

$$
g_{1}(s)=\left|\begin{array}{ll}
a_{11}(s) & a_{12}(s) \\
a_{21}(s) & a_{22}(s)
\end{array}\right|=0
$$

где

$$
\begin{aligned}
& a_{11}(s)=\left[1+\frac{\omega_{3} P_{7}}{s^{7}}+\frac{P_{8}}{s^{8}}+\underline{O}\left(\frac{1}{s^{9}}\right)\right] \omega_{3}^{n_{1}} e^{a \omega_{3} s \pi}+\frac{\phi_{13, n_{1}}(\pi, s)}{s^{8}}+\underline{O}\left(\frac{1}{s^{9}}\right), \\
& a_{12}(s)=z^{M_{6}}\left[1+\frac{\omega_{4} P_{7}}{s^{7}}+\frac{P_{8}}{s^{8}}+\underline{O}\left(\frac{1}{s^{9}}\right)\right] \omega_{4}^{n_{1}} e^{a \omega_{4} s \pi}+ \\
& +\left[1+\frac{\omega_{2} P_{7}}{s^{7}}+\frac{P_{8}}{s^{8}}+\underline{O}\left(\frac{1}{s^{9}}\right)\right] \omega_{2}^{n_{1}} e^{a \omega_{2} s \pi}+\frac{\phi_{21, n_{1}}(\pi, s)}{s^{8}}+\underline{O}\left(\frac{1}{s^{9}}\right), \\
& a_{21}(s)=\left[1+\frac{\omega_{3} P_{7}}{s^{7}}+\frac{P_{8}}{s^{8}}+\underline{O}\left(\frac{1}{s^{9}}\right)\right] \omega_{3}^{n_{2}} e^{a \omega_{3} s \pi}+\frac{\phi_{13, n_{2}}(\pi, s)}{s^{8}}+\underline{O}\left(\frac{1}{s^{9}}\right) \\
& a_{22}(s)=z^{M_{6}}\left[1+\frac{\omega_{4} P_{7}}{s^{7}}+\frac{P_{8}}{s^{8}}+\underline{O}\left(\frac{1}{s^{9}}\right)\right] \omega_{4}^{n_{2}} e^{a \omega_{4} s \pi}+
\end{aligned}
$$




$$
\begin{gathered}
+\left[1+\frac{\omega_{2} P_{7}}{s^{7}}+\frac{P_{8}}{s^{8}}+\underline{O}\left(\frac{1}{s^{9}}\right)\right] \omega_{2}^{n_{2}} e^{a \omega_{2} s \pi}+\frac{\phi_{21, n_{2}}(\pi, s)}{s^{8}}+\underline{O}\left(\frac{1}{s^{9}}\right) \\
\phi_{21, n_{p}}(\pi, s)=z^{M_{6}} \phi_{14, n_{p}}(\pi, s)+\phi_{12, n_{p}}(\pi, s), \quad p=1,2 .
\end{gathered}
$$

Раскладывая уравнение (98) по столбцам на сумму определителей, получаем

$$
g_{1}(s)=g_{1,0}(s)+\frac{g_{1,7}(s)}{s^{7}}+\frac{g_{1,8}(s)}{s^{8}}+\underline{O}\left(\frac{1}{s^{9}}\right)=0
$$

$$
\begin{aligned}
g_{1,0}(s)=\left|\begin{array}{cc}
\omega_{3}^{n_{1}} e^{a \omega_{3} s \pi} & z^{M_{6}} \omega_{4}^{n_{1}} e^{a \omega_{4} s \pi}+\omega_{2}^{n_{1}} e^{a \omega_{2} s \pi} \\
\omega_{3}^{n_{2}} e^{a \omega_{3} s \pi} & z^{M_{6}} \omega_{4}^{n_{2}} e^{a \omega_{4} s \pi}+\omega_{2}^{n_{1}} e^{a \omega_{2} s \pi}
\end{array}\right|= \\
\\
=z^{M_{6}}\left|\begin{array}{cc}
\omega_{3}^{n_{1}} & \omega_{4}^{n_{1}} \\
\omega_{3}^{n_{2}} & \omega_{4}^{n_{2}}
\end{array}\right| e^{a\left(\omega_{3}+\omega_{4}\right) s \pi}+\left|\begin{array}{cc}
\omega_{3}^{n_{1}} & \omega_{2}^{n_{1}} \\
\omega_{3}^{n_{2}} & \omega_{2}^{n_{2}}
\end{array}\right| e^{a\left(\omega_{2}+\omega_{3}\right) s \pi} ;
\end{aligned}
$$

$$
\begin{aligned}
g_{1,7}(s)=\omega_{3} P_{7}\left|\begin{array}{cc}
\omega_{3}^{n_{1}} e^{a \omega_{3} s \pi} & z^{M_{6}} \omega_{4}^{n_{1}} e^{a \omega_{4} s \pi}+\omega_{2}^{n_{1}} e^{a \omega_{2} s \pi} \\
\omega_{3}^{n_{2}} e^{a \omega_{3} s \pi} & z^{M_{6}} \omega_{4}^{n_{2}} e^{a \omega_{4} s \pi}+\omega_{2}^{n_{1}} e^{a \omega_{2} s \pi}
\end{array}\right|+ \\
+\omega_{4} P_{7}\left|\begin{array}{cc}
\omega_{3}^{n_{1}} & \omega_{4}^{n_{1}} \\
\omega_{3}^{n_{2}} & \omega_{4}^{n_{2}}
\end{array}\right| z^{M_{6}} e^{a\left(\omega_{3}+\omega_{4}\right) s \pi}+\omega_{4} P_{7}\left|\begin{array}{cc}
\omega_{3}^{n_{1}} & \omega_{2}^{n_{1}} \\
\omega_{3}^{n_{2}} & \omega_{2}^{n_{2}}
\end{array}\right| e^{a\left(\omega_{2}+\omega_{3}\right) s \pi}
\end{aligned}
$$

$$
\begin{gathered}
g_{1,8}(s)=g_{1,8,1}(s)+g_{1,8,2}(s) \\
g_{1,8,1}(s)=P_{8}\left|\begin{array}{ll}
\omega_{3}^{n_{1}} e^{a \omega_{3} s \pi} & z^{M_{6}} \omega_{4}^{n_{1}} e^{a \omega_{4} s \pi}+\omega_{2}^{n_{1}} e^{a \omega_{2} s \pi} \\
\omega_{3}^{n_{2}} e^{a \omega_{3} s \pi} & z^{M_{6}} \omega_{4}^{n_{2}} e^{a \omega_{4} s \pi}+\omega_{2}^{n_{1}} e^{a \omega_{2} s \pi}
\end{array}\right|+
\end{gathered}
$$$$
+P_{8}\left|\begin{array}{cc}
\omega_{3}^{n_{1}} & \omega_{4}^{n_{1}} \\
\omega_{3}^{n_{2}} & \omega_{4}^{n_{2}}
\end{array}\right| z^{M_{6}} e^{a\left(\omega_{3}+\omega_{4}\right) s \pi}+P_{8}\left|\begin{array}{cc}
\omega_{3}^{n_{1}} & \omega_{2}^{n_{1}} \\
\omega_{3}^{n_{2}} & \omega_{2}^{n_{2}}
\end{array}\right| e^{a\left(\omega_{2}+\omega_{3}\right) s \pi},
$$

$$
\begin{aligned}
g_{1,8,2}(s)=\left|\begin{array}{ll}
\phi_{13, n_{1}}(\pi, s) & \omega_{4}^{n_{1}} \\
\phi_{13, n_{2}}(\pi, s) & \omega_{4}^{n_{2}}
\end{array}\right| z^{M_{6}} e^{a \omega_{4} s \pi} & +\left|\begin{array}{ll}
\phi_{13, n_{1}}(\pi, s) & \omega_{2}^{n_{1}} \\
\phi_{13, n_{2}}(\pi, s) & \omega_{2}^{n_{2}}
\end{array}\right| e^{a \omega_{2} s \pi}+ \\
& +\left|\begin{array}{cc}
\omega_{3}^{n_{1}} & \phi_{21, n_{1}}(\pi, s) \\
\omega_{3}^{n_{2}} & \phi_{21, n_{2}}(\pi, s)
\end{array}\right| e^{a \omega_{3} s \pi}
\end{aligned}
$$

$$
\phi_{21, n_{p}}(\pi, s) \stackrel{(99)}{=} z^{M_{6}} \phi_{14, n_{p}}(\pi, s)+\phi_{12, n_{p}}(\pi, s) ; \quad p=1, p=2 .
$$

Из формул (10) следует, что

$$
\begin{aligned}
& \left|\begin{array}{ll}
\omega_{1}^{n_{1}} & \omega_{2}^{n_{1}} \\
\omega_{1}^{n_{2}} & \omega_{2}^{n_{2}}
\end{array}\right|=\left|\begin{array}{cc}
1^{n_{1}} & z^{n_{1}} \\
1^{n_{2}} & z^{n_{2}}
\end{array}\right|=z^{n_{2}}-z^{n_{1}}=R_{2} \neq 0 \\
& \left|\begin{array}{cc}
\omega_{2}^{n_{1}} & \omega_{3}^{n_{1}} \\
\omega_{2}^{n_{2}} & \omega_{3}^{n_{2}}
\end{array}\right|=\left|\begin{array}{ll}
z^{n_{1}} & z^{2 n_{1}} \\
z^{n_{2}} & z^{2 n_{2}}
\end{array}\right|=z^{N_{2}} R_{2}, \quad N_{2}=n_{1}+n_{2} \\
& \left|\begin{array}{cc}
\omega_{3}^{n_{1}} & \omega_{4}^{n_{1}} \\
\omega_{3}^{n_{2}} & \omega_{4}^{n_{2}}
\end{array}\right|=z^{2 N_{2}} R_{2}, \quad \ldots
\end{aligned}
$$


Подставляя формулы (102) в уравнение (100), поделим полученное уравнение на $(-1) z^{N_{2}} R_{2} e^{a\left(\omega_{4}+\omega_{3}\right) s \pi} \neq 0$ :

$$
\begin{gathered}
\widetilde{g}_{1}(s)=\widetilde{g}_{1,0}(s)+\frac{\widetilde{g}_{1,7}(s)}{s^{7}}+\frac{\widetilde{g}_{1,8,1}(s)}{s^{8}}+\frac{\widetilde{g}_{1,8,2}(s)}{s^{8}}+\underline{O}\left(\frac{1}{s^{9}}\right)=0, \\
\widetilde{g}_{1,0}(s)=e^{a\left(\omega_{2}-\omega_{4}\right) s \pi}-z^{M_{6}} z^{N_{2}} \\
\widetilde{g}_{1,7}(s)=P_{7}\left[\left(\omega_{2}+\omega_{3}\right) e^{a\left(\omega_{2}-\omega_{4}\right) s \pi}-\left(\omega_{3}+\omega_{4}\right) z^{M_{6}} z^{N_{2}}\right] ; \\
\widetilde{g}_{1,8,1}(s)=P_{8} \cdot 2 \cdot\left[e^{a\left(\omega_{2}-\omega_{4}\right) s \pi}-z^{M_{6}} z^{N_{2}}\right] \\
\widetilde{g}_{1,8,2}(s)=(-1) z^{-N_{2}} \frac{1}{R_{2}} e^{-a\left(\omega_{3}+\omega_{4}\right) s \pi} g_{1,8,2}(s) .
\end{gathered}
$$

Основное приближение уравнения (103) имеет вид

$$
\begin{gathered}
\widetilde{q}_{1,0}(s)=0 \quad \Leftrightarrow \quad e^{a\left(\omega_{2}-\omega_{4}\right) s \pi}-z^{M_{6}} z^{N_{2}}=0 \quad \Leftrightarrow \\
\Leftrightarrow \quad e^{a\left(\omega_{2}-\omega_{4}\right) s \pi}=e^{2 \pi i k} e^{\frac{2 \pi i}{8} M_{6}} e^{\frac{2 \pi i}{8} N_{2}} \Leftrightarrow s_{k, 1, \text { осн }}=\frac{2 i \widetilde{k}}{a\left(\omega_{2}-\omega_{4}\right)} \\
\tilde{k}=k+\frac{M_{6}+N_{2}}{8}, \quad k \in \mathbb{N} .
\end{gathered}
$$

Из общей теории нахождения асимптотики корней квазиполиномов вида (103) (см. [21, гл. 12; 22]) следует вид асимптотики. Справедливо следующее утверждение.

Теорема 11. Асимптотика собственных значений дифференииального оператора (1)-(8) с граничными условиями (9) в секторе 1) имеет вид

$$
\begin{gathered}
s_{k, 1}=\frac{2 i}{a\left(\omega_{2}-\omega_{4}\right)}\left[\widetilde{k}+\frac{d_{7 k, 1}}{\widetilde{k}^{7}}+\frac{d_{8 k, 1}}{\widetilde{k}^{8}}+\underline{O}\left(\frac{1}{\widetilde{k}^{9}}\right)\right], \\
\widetilde{k}=k+\frac{M_{6}+N_{2}}{8}, \quad M_{6}=\sum_{k=1}^{6} m_{k} ; \quad N_{2}=n_{1}+n_{2} ; \\
\omega_{2}=n_{1}+n_{2} ; \quad \omega_{2}-\omega_{4} \stackrel{(10)}{=} \sqrt{2} ; \quad k \in \mathbb{N} .
\end{gathered}
$$

Доказательств о. Применяя формулу Тейлора, подставляя $s_{k, 1}$ из (105) в уравнение (103), получаем

$$
\begin{aligned}
{[1+} & \left.\frac{2 \pi i d_{7 k, 1}}{\widetilde{k}^{7}}+\frac{2 \pi i d_{8 k, 1}}{\widetilde{k}^{8}}+\underline{O}\left(\frac{1}{\widetilde{k}^{9}}\right)\right] z^{M_{6}} z^{N_{2}}-z^{M_{6}} z^{N_{2}}+ \\
& +\frac{1}{\widetilde{k}^{7}} \frac{a^{7}\left(\omega_{2}-\omega_{4}\right)^{7}}{2^{7} i^{7}}\left(1+\underline{O}\left(\frac{1}{\widetilde{k}^{8}}\right)\right) P_{7}\left[\left(\omega_{2}+\omega_{3}\right) z^{M_{6}} z^{N_{2}}\left(1+\underline{O}\left(\frac{1}{\widetilde{k}^{7}}\right)\right)-\right. \\
& \left.-\left(\omega_{3}+\omega_{4}\right) z^{M_{6}} z^{N_{2}}\right]+\frac{1}{\widetilde{k}^{8}} 2 P_{8}\left[z^{M_{6}} z^{N_{2}}\left(1+\underline{O}\left(\frac{1}{\widetilde{k}^{7}}\right)\right)-z^{M_{6}} z^{N_{2}}\right]- \\
- & \left.\left.\frac{1}{\widetilde{k}^{8}} z^{-N_{2}} \frac{1}{R_{2}} e^{-a\left(\omega_{3}+\omega_{4}\right) s \pi}\right|_{s_{k, 1}} g_{1,8,2}(s)\right|_{s_{k, 1}} \frac{a^{8}\left(\omega_{2}-\omega_{4}\right)^{8}}{2^{8} i^{8}}+\underline{O}\left(\frac{1}{\widetilde{k}^{9}}\right)=0 .
\end{aligned}
$$


Приравнивая в уравнении (106) коэффициенты при $\widetilde{k}^{0}$, получаем верное равенство

$$
z^{M_{6}} z^{N_{2}}-z^{N_{6}} z^{N_{2}}=0
$$

что свидетельствует о правильности выбора асимптотики (105).

При $\widetilde{k}^{-7}$ в (106) имеем

$$
\begin{aligned}
d_{7 k, 1}=\frac{1}{2 \pi i} & \frac{P_{7} a^{7}\left(\omega_{2}-\omega_{4}\right)^{7}}{2^{7} i^{7}}\left[\left(\omega_{3}+\omega_{4}\right)-\left(\omega_{2}+\omega_{3}\right)\right]= \\
& =\frac{(-1) P_{7} a^{7}\left(\omega_{2}-\omega_{4}\right)^{8}}{2^{8} \pi} \stackrel{(97)}{=} \frac{(-1) a^{7}\left(\omega_{2}-\omega_{4}\right)^{8}}{2^{8} \pi}\left[A_{7}\left(x_{0}\right)+B_{7}\left(x_{2 n}\right)\right],
\end{aligned}
$$

откуда, применяя формулы (10), (18), и (24), находим

$$
\begin{gathered}
d_{7 k, 1}=\frac{(-1) a^{7}(\sqrt{2})^{7}}{2^{8} \pi}\left(-\frac{1}{8 a^{7}}\right)\left[\int_{x_{1 n}}^{x_{0}} q_{2}(t) d t+\int_{x_{0}}^{x_{2 n}} q_{3}(t) d t\right]= \\
=\frac{1}{128 \pi}\left[\int_{x_{1 n}}^{x_{0}} q_{2}(t) d t+\int_{x_{0}}^{x_{2 n}} q_{3}(t) d t\right] \stackrel{(5)}{=} \\
=\frac{H_{1 n}+H_{2 n}}{128 \pi} \stackrel{(5)}{=} \frac{1}{128 \pi}, \quad k=1,2,3, \ldots
\end{gathered}
$$

В предельном случае (при $n \rightarrow+\infty$, в случае, когда потенциалом изучаемого оператора является дельта-функция), получаем

$$
\begin{aligned}
\lim _{n \rightarrow+\infty} d_{7 k, 1}(n)=\lim _{n \rightarrow+\infty} \frac{1}{128 \pi}\left[\int_{x_{1 n}}^{x_{0}} q_{2}(t) d t+\int_{x_{0}}^{x_{2 n}} q_{3}(t) d t\right] \stackrel{(5)}{=} & =\frac{H_{1 n}+H_{2 n}}{128 \pi} \stackrel{(5)}{=} \frac{1}{128 \pi}
\end{aligned}
$$

При $\widetilde{k}^{-8}$ в (106) получаем

$$
\begin{aligned}
& d_{8 k, 1}=\frac{a^{8}\left(\omega_{2}-\omega_{4}\right)^{8}}{2^{8} 2 \pi i}\left[2 P_{8}(-1+1)+\right. \\
& \left.+\left.z^{-M_{6}} z^{-2 N_{2}} R_{2}^{-1} e^{-a\left(\omega_{3}+\omega_{4}\right) s \pi} g_{1,8,2}(s)\right|_{s_{k, 1, \text { осн }}}\right]= \\
& =\left.\left.\frac{z^{-M_{6}} z^{-2 N_{2}} R_{2}^{-1} a^{8}}{2^{4} 2 \pi i} e^{-a\left(\omega_{3}+\omega_{4}\right) s \pi}\right|_{s_{k, 1, \text { осн }}} g_{1,8,2}(s)\right|_{s_{k, 1, \text { осн }}}, \quad k=1,2,3, \ldots
\end{aligned}
$$

Применяя формулы (101), (96), (76), (70) и (52), формулу (108) можно переписать следующим образом:

$$
\begin{aligned}
& d_{8 k, 1}=\frac{a^{8}}{2 \pi i} \frac{z^{-M_{6}} z^{-2 N_{2}}}{16} \frac{1}{R_{2}} \times \\
& \times\left.\left[e^{a\left(\omega_{2}-\omega_{4}\right) s \pi}\left|\begin{array}{cc}
\omega_{3}^{n_{1}} & \omega_{2}^{n_{1}} \sum_{m=2}^{4} \Delta_{m 24} \\
\omega_{3}^{n_{2}} & \omega_{2}^{n_{2}} \sum_{m=2}^{4} \Delta_{m 24}
\end{array}\right| z^{M_{6}}+\left|\begin{array}{cc}
\omega_{3}^{n_{1}} & \omega_{4}^{n_{1}} \sum_{m=2}^{4} \Delta_{m 42} \\
\omega_{3}^{n_{2}} & \omega_{4}^{n_{2}} \sum_{m=2}^{4} \Delta_{m 42}
\end{array}\right|\right]\right|_{s_{k, 1 \text {, осн }}}=
\end{aligned}
$$




$$
\begin{aligned}
=\frac{a^{8}}{2 \pi i} \frac{z^{-M_{6}} z^{-2 N_{2}}}{16} \frac{1}{R_{2}}\left[z^{M_{6}} z^{N_{2}} z^{M_{6}} \sum_{m=2}^{4} \Delta_{m 24}(-1) z^{N_{2}} R_{2}+\right. \\
\left.+\sum_{m=2}^{4} \Delta_{m 42} z^{2 N_{2}} R_{2}\right]\left.\right|_{s_{k, 1, \text { осн }}},
\end{aligned}
$$

откуда, используя формулы $(77),(71)$ и $(53)$, находим $d_{8 k, 1}$ :

$$
\begin{gathered}
d_{8 k, 1}=\left.\frac{a^{8}}{32 \pi i}\left[z^{-M_{6}}\left(\Delta_{242}+\Delta_{342}+\Delta_{442}\right)-z^{-M_{6}}\left(\Delta_{224}+\Delta_{324}+\Delta_{424}\right)\right]\right|_{s_{k, 1, \text { осн }}}= \\
=\frac{a^{8}}{32 \pi i}\left\{\left[z^{-M_{6}} e^{a\left(\omega_{2}-\omega_{4}\right) s x_{1 n}} T_{7}-z^{-M_{6}} e^{-a\left(\omega_{2}-\omega_{4}\right) s x_{1 n}} T_{3}\right]+\right. \\
\quad+\left[z^{-M_{6}} e^{a\left(\omega_{2}-\omega_{4}\right) s x_{0}} R_{7}-z^{-M_{6}} e^{-a\left(\omega_{2}-\omega_{4}\right) s x_{0}} R_{3}\right]+ \\
\left.\quad+\left[z^{-M_{6}} e^{a\left(\omega_{2}-\omega_{4}\right) s x_{2 n}} V_{7}-z^{M_{6}} e^{-a\left(\omega_{2}-\omega_{4}\right) s x_{2 n}} V_{3}\right]\right\}\left.\right|_{s_{k, 1, \text { осн }}}, \quad(109) \\
T_{k} \stackrel{(\underline{77})}{=} \frac{q_{2}\left(x_{1 n}\right) G_{k}}{128 a^{8}} ; \quad R_{k} \stackrel{(68)}{=} \frac{\Delta \widetilde{q}\left(x_{0}\right) G_{k}}{128 a^{8}} ; \quad V_{k} \stackrel{(44)}{=} \frac{q_{3}\left(x_{2 n}\right) G_{k}}{128 a^{8}} \\
G_{3} \stackrel{(44)}{=} 7+5 \omega_{3}+3 \omega_{3}^{2}+\omega_{3}^{3}-\omega_{3}^{4}-3 \omega_{3}^{5}-5 \omega_{3}^{6}-7 \omega_{3}^{7}=8+8 i=8 \sqrt{2} e^{\frac{\pi i}{4}} \\
\left.G_{7}=\bar{G}_{3}=8 \sqrt{2} e^{-\frac{\pi i}{4}} ;\left.\quad e^{a\left(\omega_{2}-\omega_{4}\right) s x_{0}}\right|_{s_{k, 1, \text { осн }} \stackrel{(104)}{=} e^{2 \widetilde{k} x_{0} i} .} .110\right)
\end{gathered}
$$

Поэтому, подставив (110) в (109) и сделав необходимые преобразования, получим

$$
\begin{aligned}
& d_{8 k, 1}=\frac{a^{8}}{32 \pi i} \frac{8 \sqrt{2}}{128 a^{8}}\left\{\left[e^{-\frac{2 \pi i}{8} M_{6}} e^{\frac{-\pi i}{4}} e^{2 \widetilde{k} x_{1 n} i}-e^{\frac{2 \pi i}{8} M_{6}} e^{\frac{\pi i}{4}} e^{-2 \widetilde{k} x_{1 n} i}\right]\left(-q_{2}\left(x_{1 n}\right)\right)+\right. \\
&+ {\left[e^{-\frac{2 \pi i}{8} M_{6}} e^{\frac{-\pi i}{4}} e^{2 \widetilde{k} x_{0} i}-e^{\frac{2 \pi i}{8} M_{6}} e^{\frac{\pi i}{4}} e^{-2 \widetilde{k} x_{0} i}\right] \Delta \widetilde{q}\left(x_{0}\right)+} \\
&\left.+\left[e^{-\frac{2 \pi i}{8} M_{6}} e^{\frac{-\pi i}{4}} e^{2 \widetilde{k} x_{2 n} i}-e^{\frac{2 \pi i}{8} M_{6}} e^{\frac{\pi i}{4}} e^{-2 \widetilde{k} x_{2 n} i}\right] q_{3}\left(x_{2 n}\right)\right\}, \quad k \in \mathbb{N},
\end{aligned}
$$

откуда находим

$$
\begin{aligned}
& d_{8 k, 1}=\frac{\sqrt{2}}{256 \pi}\left\{q\left(x_{2 n}\right) \sin \left(2 \widetilde{k} x_{2 n}-\frac{\pi}{4}-\frac{\pi M_{6}}{4}\right)+\right. \\
& +\Delta \widetilde{q}\left(x_{0}\right) \sin \left(2 \widetilde{k} x_{0}-\frac{\pi}{4}-\frac{\pi M_{6}}{4}\right)- \\
& \left.-q_{2}\left(x_{1 n}\right) \sin \left(2 \widetilde{k} x_{1 n}-\frac{\pi}{4}-\frac{\pi M_{6}}{4}\right)\right\}, \quad k=1,2,3, \ldots ; \widetilde{k}=k+\frac{M_{6}+N_{2}}{8} \\
& M_{6}=\sum_{k=1}^{6} m_{k} ; \quad N_{2}=n_{1}+n_{2} ; \quad \Delta \widetilde{q}\left(x_{0}\right)=q_{2}\left(x_{0}-0\right)-q_{3}\left(x_{0}+0\right) .
\end{aligned}
$$

Формулы (107) и (111) показывают, что коэффициенты $d_{7 k, 1}$ и $d_{8 k, 1}$ асимптотического разложения (105) находятся единственным образом. Мы привели явные формулы для их вычисления, поэтому теорема 11 полностью доказана. 
Изучая аналогичным образом секторы 2), 3), . ., 8) индикаторной диаграммы (см. рисунок), можно доказать следующую теорему.

Теорема 12. В секторах 2), 3), .., 8) индикаторной диаграммы асимптотика собственных значений дифберенциального оператора (1)-(8) с граничными условиями (9) подчиняется следующему закону:

$$
\begin{gathered}
s_{k, 2}=s_{k, 1} e^{\frac{2 \pi i}{8}}, \quad s_{k, 3}=s_{k, 2} e^{\frac{2 \pi i}{8}}=s_{k, 1} e^{\frac{4 \pi i}{8}}, \ldots, \\
s_{k, m}=s_{k, m-1} e^{\frac{2 \pi i}{8}}=e^{\frac{2 \pi i}{8}(m-1)}, \quad m=1,2, \ldots, 8 .
\end{gathered}
$$

Величины $s_{k, 1}(k=1,2, \ldots, 8)$ определены формулами (105), (107), (111). При этом

$$
\lambda_{k, m}=s_{k, m}^{8}, \quad m=1,2, \ldots 8 ; \quad k=1,2,3, \ldots
$$

Конкурирующие интересы. Конкурирующих интересов не имею.

Авторская ответственность. Я несу полную ответственность за предоставление окончательной версии рукописи в печать. Окончательная версия рукописи мною одобрена.

Финансирование. Исследование выполнялось без финансирования.

\section{Библиографический список}

1. Ильин В. А. О сходимости разложений по собственным функциям в точках разрыва коэффициентов дифференциального оператора // Матем. заметки, 1977. Т. 22, № 5. C. 679-698.

2. Ильин В. А. Необходимые и достаточные условия базисности Рисса корневых векторов разрывных операторов второго порядка// Диффер. уравн., 1986. Т. 22, № 12. С. 20592071.

3. Митрохин С. И. О формулах регуляризованных следов для дифференциальных операторов второго порядка с разрывными коэффициентами // Вестн. Моск. ун-та. Сер. 1. Матем., мех., 1986. №6. С. 3-6.

4. Митрохин С. И. О формулах следов для одной краевой задачи с функциональнодифференциальным уравнением с разрывным коэффициентом // Диффер. уравн., 1986. T. 22 , № 6. С. 927-931.

5. Митрохин С. И. О некоторых спектральных свойствах дифференциальных операторов второго порядка с разрывной весовой функцией // Докл. РАН, 1997. Т. 356, № 1. С. 1315.

6. Винокуров В. А., Садовничий В. А. Асимптотика любого порядка собственных значений и собственных функций краевой задачи Штурма-Лиувилля на отрезке с суммируемым потенциалом // Диффер. уравн., 1998. Т. 34, №10. С. 1423-1426.

7. Митрохин С. И. Асимптотика собственных значений дифференциального оператора четвертого порядка с суммируемыми коэффициентами // Вестн. Моск. ун-та. Сер. 1. Матем., мех., 2009. № 3. С. 14-17.

8. Митрохин С. И. О спектральных свойствах одного дифференциального оператора с суммируемыми коэффициентами с запаздывающим аргументом // Уфимск. матем. журн., 2011. Т. 3, № 4. С. 95-115.

9. Митрохин С. И. Спектральные свойства краевых задач для функциональнодифференциального уравнения с суммируемыми коэффициентами // Диффер. уравн., 2010. Т. 46, № 8. С. 1085-1093.

10. Митрохин С. И. Асимптотика спектра периодической краевой задачи для дифференциального оператора с суммируемым потенциалом // Тр. ИММ УрО РАН, 2019. Т. 25, №1. C. 136-149. https ://doi .org/10.21538/0134-4889-2019-25-1-136-149. 
11. Савчук А. М., Шкаликов А. А. Операторы Штурма-Лиувилля с сингулярными потенциалами // Матем. заметки, 1999. Т. 66, №6. С. 897-912. https://doi.org/10.4213/ mzm1234.

12. Савчук А. М. Регуляризованный след первого порядка оператора Штурма-Лиувилля с $\delta$-потенциалом // УМH, 2000. Т. 55, №6(336). С. 155-156. https://doi.org/10.4213/ rm352.

13. Винокуров В. А., Садовничий В. А. Асимптотика собственных значений и собственных функций и формула следа для потенциала, содержащего $\delta$-функции // Диффер. уравн., 2002. Т. 38, № 6. С. 735-751.

14. Борисов Д. И. О лакунах в спектре Лапласиана в полосе с периодическим дельтавзаимодействием / Тр. ИММ УрО РАН, Т. 24, 2018. С. 46-53. https://doi.org/10. 21538/0134-4889-2018-24-2-46-53.

15. Конечная Н. Н., Сафонова Т. А., Тагирова Р. Н. Асимптотика собственных значений и регуляризованный след первого порядка оператора Штурма-Лиувилля с $\delta$-потенциалом// Вестник Северного (Арктического) федералъного университета. Сер. Eстественные науки, 2016. №1. https://doi .org/10.17238/issn2227-6572.2016.1.104.

16. Кочубей А. Н. Эллиптические операторы с граничными условиями на подмножестве меры нуль // Функи. анализ и его прил., 1982. Т. 16, № 2. С. 74-75.

17. Березин Ф. А., Фаддеев Л. Д. Замечание об уравнении Шредингера с сингулярным потенциалом // Докл. АН СССР, 1961. Т. 137, №5. С. 1011-1014.

18. Гейлер В. А., Маргулис В. А., Чучаев И. И. Потенциалы нулевого радиуса и операторы Карлемана // Сиб. матем. журн., 1995. Т. 36, № 4. С. 828-841.

19. Наймарк М. А. Линейнъе дифференциалъные операторы. М.: Наука, 1969. 528 с.

20. Митрохин С. И. Асимптотика собственных значений дифференциального оператора со знакопеременной весовой функцией // Изв. вузов. Матем., 2018. №6. С. 31-47.

21. Беллман Р., Кук К. Л. Дифференциально-разностные уравнения. М.: Мир, 1967. 548 с.

22. Садовничий В. А., Любишкин В. А. О некоторых новых результатах теории регуляризованных следов дифференциальных операторов // Диффер. уравн., 1982. Т. 18, № 1. C. 109-116. 


\title{
MSC: 34B10, 47E05
}

\section{On the asymptotics of spectrum of an even-order differential operator with a delta-function potential}

\author{
S. I. Mitrokhin \\ Lomonosov Moscow State University, \\ Research Computing Center, \\ 1, Leninskie Gory, Moscow, 119991, Russian Federation.
}

\begin{abstract}
We study a sequence of differential operators of high even order whose potentials converge to the Dirac delta-function. One of the types of separated boundary conditions is considered. At the points of potential discontinuity, it is necessary to study the conditions of gluing for the correct determination of the corresponding differential equations solutions. For large values of the spectral parameter, asymptotic solutions of differential equations are furnished by the Naimark method. The conditions of gluing are studied, the boundary conditions are investigated, the equation for the eigenvalues of the considered differential operator is derived. The method of successive approximations is used to find the asymptotics of spectrum of studied differential operators, the limit of which determines a spectrum of operator with a delta-function potential.
\end{abstract}

Keywords: differential operator, Dirac delta-function, asymptotics of solutions of differential equations, piecewise smooth potential, eigenvalues, asymptotics of the spectrum.

Received: $15^{\text {th }}$ July, 2020 / Revised: $23^{\text {rd }}$ November, $2021 /$

Accepted: $6^{\text {th }}$ December, $2021 /$ First online: $29^{\text {th }}$ December, 2021

Competing interests. I have no competing interests.

Author's Responsibilities. I take full responsibility for submit the final manuscript to print. I approved the final version of the manuscript.

Funding. This research received no external funding.

\section{Research Article}

(C) Authors, 2021

(C) Samara State Technical University, 2021 (Compilation, Design, and Layout)

ㄱ (ㅇ)(i) The content is published under the terms of the Creative Commons Attribution 4.0 International License (http://creativecommons.org/licenses/by/4.0/)

Please cite this paper in press as:

Mitrokhin S. I. On the asymptotics of spectrum of an even-order differential operator with a delta-function potential, Vestn. Samar. Gos. Tekhn. Univ., Ser. Fiz.-Mat. Nauki [J. Samara State Tech. Univ., Ser. Phys. Math. Sci.], 2021, vol. 25, no. 4, pp. 634-662. https://doi.org/10.14498/vsgtu1798 (In Russian).

\section{Author's Details:}

Sergey I. Mitrokhin (10) https://orcid.org/0000-0003-1896-0563

Cand. Phys. \& Math. Sci., Associate Professor; Senior Researcher; Research Computing Center; e-mail:mitrokhin-sergey@yandex.ru 


\section{References}

1. Il'in V. A. Convergence of eigenfunction expansions at points of discontinuity of the coefficients of a differential operator, Math. Notes, 1977, vol. 22, no. 5, pp. 870-882. https:// doi.org/10.1007/BF01098352.

2. Il'in V. A. Necessary and sufficient conditions for being a Riesz basis of root vectors of second-order discontinuous operators, Differ. Uravn., 1986, vol. 22, no. 12, pp. 2059-2071 (In Russian).

3. Mitrokhin S. I. Formulas for the regularized traces of the second order differential operators with discontinuous coefficients, Mosc. Univ. Math. Bull., 1986, vol. 41, no. 6, pp. 1-5.

4. Mitrokhin S. I. Trace formulas for a boundary value problem with a functional-differential equation with a discontinuous coefficient, Differ. Uravn., 1986, vol. 22, no. 6, pp. 927-931 (In Russian).

5. Mitrokhin S. I. On some spectral properties of second-order differential operators with a discontinuous positive weight function, Dokl. Akad. Nauk, 1997, vol.356, no.1, pp. 13-15 (In Russian).

6. Vinokurov V. A., Sadovnichii V. A. Arbitrary-order asymptotics of the eigenvalues and eigenfunctions of the Sturm-Liouville boundary value problem on an interval with integrable potential, Differ. Equ., 1998, vol. 34, no. 10, pp. 1425-1429.

7. Mitrokhin S. I. The asymptotics of the eigenvalues of a fourth order differential operator with summable coefficients, Mosc. Univ. Math. Bull., 2009, vol. 64, no. 3, pp. 102-104.

8. Mitrokhin S. I. On spectral properties of a differential operator with summable coefficients with a retarded argument, Ufimsk. Mat. Zh., 2011, vol. 3, no. 4, pp. 95-115 (In Russian).

9. Mitrokhin S. I. Spectral properties of boundary value problems for functional-differential equations with integrable coefficients, Differ. Equ., 2010, vol.46, no.8, pp. 1095-1103. https://doi.org/10.1134/S0012266110080033.

10. Mitrokhin S. I. Asymptotics of the spectrum of a periodic boundary value problem for a differential operator with a summable potential, Trudy Inst. Mat. Mekh. UrO RAN, 2019, vol.25, no.1, pp. 136-149 (In Russian). https://doi.org/10.21538/ 0134-4889-2019-25-1-136-149.

11. Savchuk A. M., Shkalikov A. A. Sturm-Liouville operators with singular potentials, Math. Notes, 1999, vol.66, no.6, pp. 741-753. https://doi.org/10.1007/BF02674332.

12. Savchuk A. M. First-order regularised trace of the Sturm-Liouville operator with $\delta$ potential, Russian Math. Surveys, 2000, vol.55, no.6, pp. 1168-1169. https://doi.org/ 10.1070/rm2000v055n06ABEH000352.

13. Vinokurov V. A., Sadovnichii V. A. The asymptotics of eigenvalues and eigenfunctions and a trace formula for a potential with delta functions, Differ. Equ., 2002, vol. 38, no. 6, pp. 772-789. https://doi.org/10.1023/A:1020302110566.

14. Borisov D. I. Gaps in the spectrum of the Laplacian in a strip with periodic delta interaction, Proc. Steklov Inst. Math. (Suppl.), 2019, vol. 305 (suppl. 1), pp. S16-S23. https://doi.org/ 10.1134/S0081543819040047.

15. Konechnaya N. N., Safonova T. A., Tagirova R. N. Asymptotics of the eigenvalues and regularized trace of the first-order Sturm-Liouville operator with $\delta$-potential, Vestnik of Northern (Arctic) Federal University. Ser. Natural Science, 2016, no. 1, pp. 104-113 (In Russian). https://doi.org/10.17238/issn2227-6572.2016.1.104.

16. Kochubei A. N. Elliptic operators with boundary conditions on a subset of measure zero, Funct. Anal. Appl., 1982, vol.16, no. 2, pp. 137-139. https://doi.org/10.1007/ $\mathrm{BF} 01081632$.

17. Berezin F. A., Faddeev L. D. A remark on Schrödinger's equation with a singular potential, Sov. Math., Dokl., 1961, vol. 2, no. 5, pp. 372-375.

18. Geiler V. A., Margulis V. A., Chuchaev I. I. Potentials of zero radius and Carleman operators, Siberian Math. J., 1995, vol.36, no.4, pp. 714-726. https://doi.org/10.1007/ BF02107328. 
19. Naimark M. A. Lineinye differentsial'nye operatory [Linear Differential Operators]. Moscow, Nauka, 1969, 528 pp. (In Russian)

20. Mitrokhin S. I. Asymptotics of eigenvalues of differential operator with alternating weight function, Russian Math. (Iz. VUZ), 2018, vol.62, no.6, pp. 27-42. https://doi.org/10. 3103/S1066369X1806004X.

21. Bellman R., Cooke K. L. Differential-Difference Equations, Mathematics in Science and Engineering, vol. 6. New York, London, Academic Press, 1963, xvi+462 pp.

22. Sadovnichii V. A., Lyubishkin V. A. Some new results of the theory of regularized traces of differential operators, Differ. Uravn., 1982, vol. 18, no. 1, pp. 109-116 (In Russian). 